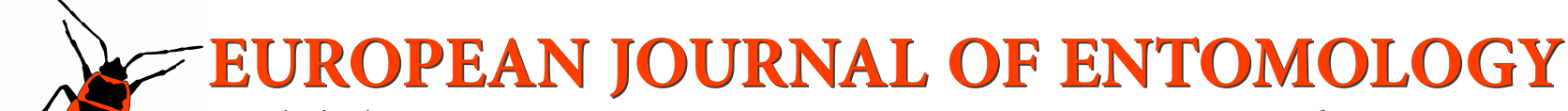 \\ ISSN (online): 1802-8829 \\ http://www.eje.cz \\ Eur. J. Entomol. 117: 490-503, 2020 \\ doi: 10.14411/eje.2020.052 \\ ORIGINAL ARTICLE
}

\section{Distinct barcodes for the Cereal leaf beetles Oulema melanopus and Oulema duftschmidi (Coleoptera: Chrysomelidae), two syntopical sibling species}

\author{
Julie LeROY ${ }^{1}$, Jean-David CHAPELIN-VISCARDI ${ }^{1}$, Guénaëlle GENSON ${ }^{2}$, Julien HARAN² ${ }^{2}$ Éric PIERRE ${ }^{2}$ \\ and JEAN-CLAUde STREITO ${ }^{2}$ \\ ${ }^{1}$ Laboratoire d'Éco-Entomologie, 5 rue Antoine Mariotte, 45000 Orléans, France; \\ e-mails: leroy@laboratoireecoentomologie.com, chapelinviscardi@laboratoireecoentomologie.com \\ ${ }^{2}$ CBGP, INRAE, CIRAD, IRD, Institut Agro, Univ Montpellier, Montpellier, France; e-mails: guenaelle.genson@inrae.fr, \\ julien.haran@cirad.fr, eric.pierre@inrae.fr, jean-claude.streito@inrae.fr
}

Key words. Coleoptera, Chrysomelidae, Oulema, cereal leaf beetles, DNA barcode, COI, identification, crop pests, Europe

\begin{abstract}
Oulema melanopus (Linnaeus, 1758) and Oulema duftschmidi (Redtenbacher, 1874) (Coleoptera: Chrysomelidae) are two native West Palaearctic species developing on various cultivated and wild grasses. Along with $O$. obscura they are considered to be secondary pests of cereal crops. However, local outbreaks have been recorded recently and their status as secondary pests may evolve, especially as the use of broad-spectrum insecticides is now greatly reduced. Oulema melanopus and $O$. duftschmidi are considered to be sibling species. They are morphologically very close and difficult to distinguish from each other, which makes it difficult to study them. We tested the reliability of the standard barcode fragment $(C O I)$ for distinguishing between these species. A total of 92 samples of the two species, covering the majority of their natural range, was sequenced for the barcode fragment and inter- and intraspecific genetic distances were estimated. Our results confirm those of Bezděk \& Baselga (2015, Acta Entomol. Mus. Nat. Prag. 55: 273-304) in that this marker cannot differentiate between all the species of the Oulema melanopus complex, which in the Mediterranean basin contains several described and possibly some undescribed cryptic species. However, this marker may be useful in an agricultural context in areas where only O. melanopus and O. duftschmidi occur (such as in cereal crops in France) where it can be used to reliably and rapidly separate all stages of these two taxa and can therefore help in studying their ecology and dynamics.
\end{abstract}

\section{INTRODUCTION}

Cereal leaf beetles belong to Coleoptera of the subfamily Criocerinae within the family Chrysomelidae and their larvae feed and develop on various cultivated or wild grasses (Venturi, 1942; Jolivet, 1997). Nine species are recognized in France, one belonging to the genus Lema [Lema cyanella (Linnaeus, 1758)] and 8 to the genus Oulema [Oulema duftschmidi (Redtenbacher, 1874), O. erichsonii (Suffrian, 1841), O. obscura (Stephens, 1831), O. hoffmannseggii (Lacordaire, 1845), O. melanopus (Linnaeus, 1758), O. rufocyanea (Suffrian, 1847), O. septentrionis (Weise, 1880) and $O$. tristis (Herbst, 1786)]. The genus Oulema comprises about 130 species worldwide, of which 19 are Nearctic and 21 Palearctic. Currently, 11 species are known from Europe (Bezděk \& Schmitt, 2017; Rilet et al., 2003), among which two new species were recently described from Italy (Oulema mauroi Bezděk \& Baselga, 2015) and Spain (Oulema verae Bezděk \& Baselga, 2015). Only two species of Oulema are frequently cited as cereal pests in the Palearctic region: O. melanopus and O. obscura (Bala- chowsky \& Mesnil, 1935; Bonnemaison, 1962; Labeyrie, 1963; Chambon et al., 1983; ACTA, 2016). The history of the taxonomy of $O$. obscura is very confusing: early works mention O. obscura as Lema lichenis Weise, 1882 or Lema lichenis Voet, 1806 (an invalid name according to White, 1981), then as O. gallaeciana (Heyden, 1870), before it was synonymized with $O$. obscura (Stephens, 1831) (Cox, 2000; Bezděk \& Schmitt, 2017). Labeyrie (1963) further cites $O$. tristis as a pest, as does Feytaud (1924). With the exception of $O$. tristis, which has not been reported on crops since then, three species of Oulema are regularly observed in crops and are likely to damage them in France and Europe: O. obscura, O. melanopus, but also $O$. duftschmidi, which was confused with the previous species until 1989 and is still not included in plant protection manuals. $O$. melanopus and $O$. duftschmidi are considered to be sibling species, as they are very similar both in their internal (genitalia) and external morphology. Their identification requires the dissection of the flagellum located in the penis of the males (Berti, 1989; Bezděk \& Baselga, 
2015; Chapelin-Viscardi \& Maillet-Mezeray, 2015; Leroy \& Chapelin-Viscardi, 2018), which makes their study particularly difficult for non-specialists, especially as it is not possible to identify females and the immature stages. The existence of both species in France was reported by Berti (1989), who provides reliable morphological criteria for identifying them (Fig. 2) and reports the existence of specimens of $O$. duftschmidi identified as $O$. melanopus in the collections of the French National Museum of Natural History (MNHN, Paris). Berti (1989) also states that both species are sympatric and widely distributed in France. More recently, Bezděk \& Baselga (2015) revised the Oulema melanopus species complex in Western Europe. They recognize five species, including two new ones and review the taxonomy of the group.

Oulema melanopus and $O$. duftschmidi can damage crops, especially the larvae that feed on leaves of cereal plants (Philips et al., 2011). These sibling species could potentially harm cereal crops in France (Bonnemaison, 1962; Labeyrie, 1963; Anglade et al., 1976; Chambon et al., 1983; ACTA, 2016 etc.) and other European countries (Labeyrie, 1963): Romania (Knechtel \& Monolache, 1936), Hungary (Sajó, 1893), Spain (Urquijo, 1940), Greece (Pélécassis, 1951) and Italy (Bechini et al., 2013). Most of these publications refer only to $O$. melanopus whereas O. duftschmidi may be also involved (Chapelin-Viscardi \& Maillet-Mezeray, 2015). Recently, high population densities of larvae of cereal leaf beetles were recorded in various parts of mainland France, such as the Ille-et-Vilaine, Loiret and Allier departments (pers. obs.). In addition, extreme climatic events, which are becoming increasingly frequent, are conducive to pest outbreaks, particularly of species that are highly dependent on the climate, such as cereal leaf beetles (Guppy \& Harcourt, 1978; Olfert \& Weiss, 2006; Bechini et al., 2013). The greatly reduced use of neonicotinoid insecticides, and more generally that of pesticides, along with the increase in organic farming in Europe, could also provide suitable conditions for future outbreaks of these pests. Their status could shift from secondary to major pests, as has occurred in the United States and Asia (Philips et al., 2011).

Due to the impossibility of identifying the larvae of these species little is known about the life history traits and relative abundance of these two species. Preliminary agricultural monitoring indicates that $O$. duftschmidi is the more common in several French agricultural landscapes (Chapelin-Viscardi \& Maillet-Mezeray, 2015). These surveys also reveal that the flight activity of the adults of both these species is synchronous, which indicates simultaneous larval development. A more recent biogeographical study provides clear evidence that both species are sympatric and coexist throughout France (Leroy \& Chapelin-Viscardi, 2018).

In order to better understand the structure of the $O$. melanopus/O. duftschmidi species complex it is crucial to have a reliable and routine method for identifying all the development stages of the species. Kubisz et al. (2012) used the standard barcode fragment of the mitochondrial
COI gene (Hebert et al., 2003a) to successfully distinguish between and identify several species of Criocerinae in the genus Crioceris. Similarly, Bezděk \& Baselga (2015) use this DNA fragment for identifying European species of Oulema, but their results, based on a small number of specimens, indicate that the COI barcode is not appropriate for the molecular identification of these species as there are discrepancies between the species boundaries revealed by morphology and DNA barcodes. In this study the effectiveness of this gene for identifying the species of Oulema feeding on cereals in Europe is re-evaluated using a larger number of specimens.

\section{MATERIALS AND METHODS}

\section{Sampling and morphological identification}

Specimens were collected between 2005 and 2017 in France, Portugal (Madeira), Greece (Crete), Spain and Italy (Table 1 and Fig. 1). At each site sampled, one to three adults or larvae were collected and killed directly in $96.5 \%$ ethanol. The adult specimens were identified to species, based on external morphological characters (Warchałowski, 2003; Bezděk \& Mlejnek, 2016), except for specimens belonging to the Oulema melanopus/duftschmidi species pair (Fig. 2) for which the identification was based on the structure of dissected male genitalia as only by examination of the flagellum can the two species in this complex be reliably separated (Chapelin-Viscardi \& Maillet-Mezeray, 2015). O. duftschmidi has a thin, elongated flagellum (Fig. 2d, f) whereas $O$. melanopus has a short, stocky flagellum (Fig. 2e, g) (Bukejs \& Ferenca, 2010; Bezděk \& Baselga, 2015). For this reason, only males were used to validate our DNA sequences for $O$. melanopus and $O$. duftschmidi. Three species of Oulema were sequenced, including 92 male specimens belonging to the O. melanopus/duftschmidi complex (44O. melanopus ô, $48 O$. duftschmidi ${ }^{\lambda}$ ) and 43 specimens of both sexes of $O$. obscura. To account for these species in an evolutionary context and validate our means of identification, 25 additional specimens of 8 different species belonging to the subfamily Criocerinae were also sampled. The species Epitrix pubescens (Chrysomelidae: Galerucinae) was used as an outgroup to root the phylogenetic analysis reported below. After validation of the barcoding method, we tested the molecular identification of 17 females and 7 larvae of the $O$. melanopus/duftschmidi complex. As a result, the sample for molecular analyses included a total of 185 specimens (Table 1).

\section{DNA sequencing and analysis of sequences}

The extraction and amplification protocol was that used by Streito et al. (2018): extraction of the total genomic DNA was carried out in a non-destructive manner, on whole specimens, using the DNeasy 96 Blood \& Tissue extraction kit (Qiagen, Hilden, Germany) following the manufacturer's protocol. The standard barcode fragment (Hebert et al., 2003a) was amplified using a mixture of tailed primers (based on Cruaud et al., 2010; Germain et al., 2013 and Ivanova et al., 2007) (Table 2). PCRs were carried out in $25 \mu \mathrm{l}$ of reagents with $2 \mu \mathrm{l}$ of matrix DNA, $0.07 \mathrm{mM}$ of each primer, $2.5 \mathrm{mM}$ of $\mathrm{MgCl}_{2}, 0.05 \mathrm{mM}$ of dNTPs and 0.025 U/ $\mu$ l Dreamtaq DNA Polymerase (Thermo Scientific, Waltham, USA). The PCR conditions used were $94^{\circ} \mathrm{C}$ for $3 \mathrm{~min}$ followed by 5 cycles at $94^{\circ} \mathrm{C}$ for $30 \mathrm{~s}, 45^{\circ} \mathrm{C}$ for $40 \mathrm{~s}, 72^{\circ} \mathrm{C}$ for $60 \mathrm{~s}$, then 35 cycles at $94^{\circ} \mathrm{C}$ for $30 \mathrm{~s}, 51^{\circ} \mathrm{C}$ for $40 \mathrm{~s}, 72^{\circ} \mathrm{C}$ for $60 \mathrm{~s}$, with a final extension phase at $72^{\circ} \mathrm{C}$ for $10 \mathrm{~min}$. PCR products were purified, then sequenced directly by Eurofins MWG Operon according to their protocol using M13 sequencing primers (M13F and M13R). Forward and reverse overlapping strands were assembled 
Table 1. List of sequenced specimens and the sequence accession numbers available in Bold and GenBank. For presentation purposes, COI sequences of specimens highlighted in grey were not included in the phylogenetic trees (Figs 3 and S1).

\begin{tabular}{|c|c|c|c|c|c|c|c|c|c|}
\hline Species & Specimens & $\begin{array}{l}\text { Phylogenetic L } \\
\text { tree codes }\end{array}$ & $\begin{array}{l}\text { Life stage } \\
\quad(\text { sex })\end{array}$ & Country & Locality & $\begin{array}{l}\text { Geographic coordinates } \\
\text { Lon./Lat., decimal degr. }\end{array}$ & $\begin{array}{l}\text { Sampling } \\
\text { date }\end{array}$ & BOLD ID & $\begin{array}{l}\text { GenBank } \\
\text { Access no. }\end{array}$ \\
\hline \multirow{5}{*}{$\begin{array}{l}\text { Crioceris asparagi } \\
(\text { L., 1758) }\end{array}$} & CCOC09462_0101 & - & adult & France & La Chapelle-sur-Loire & $47.238952 / 0.185079$ & $15 / 07 / 2010$ & BCELB001-19 & MT456383 \\
\hline & CCOC09463_0101 & - & adult & France & La Chapelle-sur-Loire & $47.238952 / 0.185079$ & $15 / 07 / 2010$ & BCELB002-19 & MT456384 \\
\hline & CCOC09464_0101 & - & adult & France & La Chapelle-sur-Loire & $47.238952 / 0.185079$ & $15 / 07 / 2010$ & BCELB003-19 & MT456382 \\
\hline & CCOC00752_0101 & C007520101 & adult & France & La Chapelle-sur-Loire & $47.249645 / 0.210105$ & $10 / 07 / 2005$ & BCELB004-19 & MT456385 \\
\hline & CCOC02373_0101 & C023730101 & adult & France & Vernou-sur-Brenne & $47.400450 / 0.863481$ & $19 / 05 / 2007$ & BCELB005-19 & MT456386 \\
\hline \multirow{2}{*}{$\begin{array}{l}\text { Crioceris bicruciata } \\
\text { (Sahlberg, 1823) }\end{array}$} & JSTR00373_0101 & R003730101 & adult & Greece & Askinou (Crete) & $35.291880 / 24.178090$ & $25 / 05 / 2013$ & BCELB006-19 & MT456388 \\
\hline & JSTR00373_0103 & $\mathrm{R} 003730103$ & adult & Greece & Askinou (Crete) & $35.291880 / 24.178090$ & $25 / 05 / 2013$ & BCELB007-19 & MT456387 \\
\hline \multirow{5}{*}{$\begin{array}{l}\text { Crioceris duodecim- } \\
\text { punctata (L., 1758) }\end{array}$} & CCOC00757_0101 & - & adult & France & La Chapelle-sur-Loire & $47.249645 / 0.210105$ & $13 / 07 / 2005$ & BCELB008-19 & MT456390 \\
\hline & CCOC02404_0101 & - & adult & France & La Chapelle-sur-Loire & $47.248332 / 0.194144$ & $13 / 05 / 2007$ & BCELB009-19 & MT456391 \\
\hline & JSTR01304_0101 & - & adult & France & Villandry & $47.340936 / 0.500805$ & $18 / 06 / 2012$ & BCELB010-19 & MT456392 \\
\hline & CCOC00555_0101 & C005550101 & adult & France & Montauroux & $43.617652 / 6.807396$ & $18 / 09 / 2005$ & BCELB011-19 & MT456389 \\
\hline & CCOC01914_0101 & C019140101 & adult & France & Saint-Martin-en-Ré & $46.198628 /-1.344658$ & $12 / 08 / 2007$ & BCELB012-19 & MT456393 \\
\hline \multirow{4}{*}{$\begin{array}{l}\text { Crioceris } \\
\text { paracenthesis } \\
(\mathrm{L} ., 1767)\end{array}$} & JSTR01291_0102 & - & adult & France & Saint-Raphaël & $43.417100 / 6.859020$ & $10 / 07 / 2005$ & BCELB013-19 & MT456395 \\
\hline & JSTR01296_0101 & - & adult & France & Sainte-Croix-de-Quintillargue & $43.775419 / 3.907606$ & 07/05/2005 & BCELB014-19 & MT456396 \\
\hline & JSTR01291_0101 & R012910101 & adult & France & Saint-Raphaël & $43.417100 / 6.859020$ & $10 / 07 / 2005$ & BCELB015-19 & MT456394 \\
\hline & JSTR01294_0101 & R012940101 & adult & France & Mireval & $43.531030 / 3.802800$ & $30 / 04 / 2005$ & BCELB016-19 & MT456397 \\
\hline \multirow{2}{*}{$\begin{array}{l}\text { Lilioceris lilii } \\
\text { (Scopoli, 1763) }\end{array}$} & JSTR01305_0101 & R013050101 & adult & France & Montlouis-sur-Loire & $47.370624 / 0.837422$ & $26 / 04 / 2012$ & BCELB024-19 & MT456406 \\
\hline & JSTR01305_0102 & $\mathrm{R} 013050102$ & adult & France & Montlouis-sur-Loire & $47.370624 / 0.837422$ & $26 / 04 / 2012$ & BCELB025-19 & MT456407 \\
\hline \multirow{3}{*}{$\begin{array}{l}\text { Lema aenea } \\
\text { (Lacordaire, 1845) }\end{array}$} & JSTR01223_0101 & - & adult & France & Salazie (Reunion Island) & $-21.052480 / 55.5257602$ & $29 / 03 / 2014$ & BCELB017-19 & MT456399 \\
\hline & JSTR00629_0101 & R006290101 & adult & France $S$ & Saint-Benoît (Reunion Island) & $-21.096460 / 55.653440$ & $25 / 03 / 2014$ & BCELB018-19 & MT456400 \\
\hline & JSTR00629_0102 & $\mathrm{R} 006290102$ & adult & France $S$ & Saint-Benoît (Reunion Island) & $-21.096460 / 55.653440$ & $25 / 03 / 2014$ & BCELB019-19 & MT456401 \\
\hline \multirow{3}{*}{$\begin{array}{l}\text { Lema borboniae } \\
\text { (Jolivet, 1979) }\end{array}$} & JSTR01222_0101 & - & adult & France & Salazie (Reunion Island) & $-21.052480 / 55.525760$ & $29 / 03 / 2014$ & BCELB020-19 & MT456402 \\
\hline & JSTR00629_1301 & R006291301 & adult & France $S$ & Saint-Benoît (Reunion Island) & $-21.096460 / 55.653440$ & $25 / 03 / 2014$ & BCELB021-19 & MT456403 \\
\hline & JSTR00629_1302 & $\mathrm{R} 006291302$ & adult & France $\subseteq$ & Saint-Benoît (Reunion Island) & $-21.096460 / 55.653440$ & $25 / 03 / 2014$ & BCELB022-19 & MT456404 \\
\hline Lema cyanella (L., 1758) & ) JSTR04552_0201 & R045520201 & adult (우) & Spain & Villafranca de Ebro & $41.540200 /-0.565540$ & $6 / 8 / 2017$ & BCELB023-19 & MT456405 \\
\hline & $\mathrm{JDCH} 00002 \_0302$ & $-\quad$ & adult $\left(\delta^{\lambda}\right)$ & France & Mirecourt & $48.286220 / 6.106403$ & $25 / 03 / 2014$ & BCELB026-19 & MT456451 \\
\hline (Ruetdenbacher, 1874) & JDCH00003_0302 & - & adult $(\widehat{0})$ & France & Gannat & $46.100167 / 3.199103$ & $24 / 04 / 2014$ & BCELB027-19 & MT456452 \\
\hline & JDCH00003_0303 & - & adult $(\hat{O})$ & France & Gannat & $46.100167 / 3.199103$ & $24 / 04 / 2014$ & BCELB028-19 & MT456453 \\
\hline & JDCH00004_0302 & - & adult $(\hat{0})$ & France & Houville-la-Branche & $48.447991 / 1.625547$ & $18 / 07 / 2014$ & BCELB029-19 & MT456454 \\
\hline & JDCH00005_0302 & - & adult $(\hat{0})$ & France & Treffendel & $48.037544 /-1.983165$ & 06/06/2014 & BCELB030-19 & MT456455 \\
\hline & JDCH00006_0302 & - & adult $(\widehat{\partial})$ & France & Saint-Aubin-du-Désert & $48.322195 /-0.203475$ & $15 / 05 / 2014$ & BCELB031-19 & MT456456 \\
\hline & JDCH00009_0302 & - & adult $(\hat{\partial})$ & France & Querrieu & $49.945034 / 2.424406$ & $25 / 07 / 2014$ & BCELB032-19 & MT456457 \\
\hline & JDCH00010_0302 & - & adult $(\widehat{0})$ & France & Thiverval-Grignon & $48.846643 / 1.951940$ & 01/06/2014 & BCELB033-19 & MT456458 \\
\hline & JDCH00015_0302 & - & adult $\left({ }^{\lambda}\right)$ & France & Orléans & - & $15 / 06 / 2013$ & BCELB034-19 & MT456459 \\
\hline & JDCH00018_0302 & - & adult $(\widehat{\partial})$ & France & Tilloy-lès-Mofflaines & $50.277301 / 2.806932$ & $15 / 05 / 2014$ & BCELB035-19 & MT456460 \\
\hline & JDCH00018_0303 & - & adult $\left({ }^{\lambda}\right)$ & France & Tilloy-lès-Mofflaines & $50.277301 / 2.806932$ & $15 / 05 / 2014$ & BCELB036-19 & MT456461 \\
\hline & JDCH00020_0302 & - & adult $(\widehat{\partial})$ & Portugal $\mathrm{F}$ & Porto Santo Island (Madeira) & $33.083000 /-16.334400$ & 04/04/2015 & BCELB038-19 & MT456463 \\
\hline & JDCH00021_0301 & - & adult $\left(\partial^{\prime}\right)$ & Portugal $\mathrm{F}$ & Porto Santo Island (Madeira) & $33.083000 /-16.334400$ & $04 / 0$ & BCELB039-19 & MT456464 \\
\hline & JDCH00025_0302 & - & adult $\left(0^{2}\right)$ & France & Montargis & $47.995572 / 2.730073$ & $12 / 07 / 2015$ & BCELB040-19 & MT456465 \\
\hline & JDCH00026_0302 & - & adult $\left(\partial^{\lambda}\right)$ & Greece & Chania (Crete) & $35.476613 / 23.933068$ & $31 / 05 / 2016$ & BCELB041-19 & MT456466 \\
\hline & JSTR01261_0101 & - & adult $(0)$ & France & Neuville-Vitasse & $50.255900 / 2.809390$ & $15 / 05 / 2014$ & BCELB037-19 & MT456462 \\
\hline & JSTR03883_0102 & - & adult $\left({ }^{\lambda}\right)$ & Italy & Collemeto & $40.203419 / 18.093920$ & $16 / 11 / 2016$ & BCELB042-19 & MT456467 \\
\hline & JDCH00002_0301 & H000020301 & adult $(\widehat{\partial})$ & France & Mirecourt & $48.286220 / 6.106403$ & $25 / 03 / 2014$ & BCELB043-19 & MT456468 \\
\hline & JDCH00003_0301 & $\mathrm{H} 000030301$ & adult $\left({ }^{\lambda}\right)$ & France & Gannat & $46.100167 / 3.199103$ & $24 / 04 / 2014$ & BCELB044-19 & MT456469 \\
\hline & JDCH00004_0301 & H000040301 & adult $(\precsim)$ & France & Houville-la-Branche & $48.447991 / 1.625547$ & $18 / 07 / 2014$ & BCELBO & MT456470 \\
\hline & JDCH00005_0301 & $\mathrm{H} 000050301$ & adult $(\precsim)$ & France & Treffendel & $48.037544 /-1.983165$ & $06 / 06 / 2014$ & BCELB046-19 & MT456471 \\
\hline & JDCH00006_0301 & H000060301 & adult $(\precsim)$ & France & Saint-Aubin-du-Désert & $48.322195 /-0.203475$ & $15 / 05 / 2014$ & BCELB047-19 & MT456472 \\
\hline & JDCH00007_0301 & $\mathrm{H} 000070301$ & adult $(\widehat{\partial})$ & France & Cossé-le-Vivien & $47.947795 /-0.921883$ & $15 / 05 / 2014$ & BCELB048-19 & MT456473 \\
\hline & JDCH00008_0301 & H000080301 & adult $(\precsim)$ & France & Beugnâtre & $50.129035 / 2.874760$ & $14 / 05 / 2014$ & BCELBO4 & MT456474 \\
\hline & JDCH00009_0301 & H000090301 & adult $(\AA)$ & France & Querrieu & $49.945034 / 2.424406$ & $25 / 07 / 2014$ & BCELB050-19 & MT456475 \\
\hline & JDCH00010_0301 & H000100301 & adult $(\widehat{\partial})$ & France & Thiverval-Grignon & $48.846643 / 1.951940$ & $01 / 06 / 2014$ & BCELB051-19 & MT456476 \\
\hline & JDCH00012_0301 & H000120301 & adult $(\hat{0})$ & France & Bruxerolles & $46.611001 / 0.386810$ & $18 / 05 / 2014$ & BCELB052-19 & MT456477 \\
\hline & JDCH00013_0301 & H000130301 & adult $(\widehat{\partial})$ & France & Mouzeuil-Saint-Martin & $46.465235 /-0.983798$ & $15 / 06 / 2014$ & BCELB053-19 & MT456478 \\
\hline & JDCH00014_0301 & H000140301 & adult $(\precsim)$ & France & Saint-Priest & $45.687722 / 4.967944$ & $30 / 06 / 2014$ & BCELB054-19 & MT456479 \\
\hline & JDCH00015_0301 & H000150301 & adult $(\hat{O})$ & France & Orléans & - & $15 / 06 / 2013$ & BCELB055-19 & MT456432 \\
\hline & JDCH00016_0301 & $\mathrm{H} 000160301$ & adult $(ð)$ & France & Parcieux & $45.917306 / 4.835083$ & $07 / 07 / 2014$ & BCELB056-19 & MT456433 \\
\hline & JDCH00017_0302 & $\mathrm{H} 000170302$ & adult $(ð)$ & France & Plaisir & $48.793955 / 1.942767$ & $03 / 05 / 2014$ & BCELB057-19 & MT456434 \\
\hline & JDCH00018_0301 & H000180301 & adult $(\precsim)$ & France & Tilloy-lès-Mofflaines & $50.277301 / 2.806932$ & $15 / 05 / 2014$ & BCELB058-19 & MT456435 \\
\hline & JDCH00019_0301 & H000190301 & adult $(ð)$ & France & Neuville-Vitasse & $50.255900 / 2.809400$ & $23 / 07 / 2014$ & BCELB059-19 & MT456436 \\
\hline & JDCH00020_0301 & H000200301 & adult ( & Portugal $\mathrm{F}$ & orto Santo Island (Madei & $33.083000 /-16.334400$ & $04 / 04 / 2015$ & BCELB064-19 & MT456441 \\
\hline & JDCH00021_0302 & $\mathrm{H} 000210302$ & adult ( & Portugal $\mathrm{F}$ & rto Santo Island (Madei & $33.083000 /-16.334400$ & 04/04/2015 & BCELB065-19 & MT456450 \\
\hline & JDCH00022_0301 & H000220301 & adult $(\precsim)$ & France & Lectoure & $43.933604 / 0.623621$ & $12 / 05 / 2015$ & BCELB066-19 & MT456449 \\
\hline & JDCH00024_0301 & $\mathrm{H} 000240301$ & adult $(\precsim)$ & France & Ladon & $48.004543 / 2.533953$ & $05 / 07 / 2015$ & BCELB067-19 & MT456448 \\
\hline & JDCH00025_0301 & H000250301 & adult $(\precsim)$ & France & Montargis & $47.995572 / 2.730073$ & $12 / 07 / 2015$ & BCELB068-19 & MT456447 \\
\hline & JDCH00026_0301 & $\mathrm{H} 000260301$ & adult $(ð)$ & Greece & Chania (Crete) & $35.476613 / 23.933068$ & $31 / 05 / 2016$ & BCELB069-19 & MT456446 \\
\hline & JDCH00026_0303 & $\mathrm{H} 000260303$ & adult $(\precsim)$ & Greece & Chania (Crete) & $35.476613 / 23.933068$ & $31 / 05 / 2016$ & BCELB070-19 & MT456445 \\
\hline & JSTR00666_0102 & $\mathrm{R} 006660102$ & adult $(ð)$ & France & Arles & $43.510278 / 4.781944$ & $27 / 04 / 2014$ & BCELB060-19 & MT456437 \\
\hline & JSTR01258_0101 & R012580101 & adult $(\widehat{\partial})$ & France & Lorgies & $50.554260 / 2.793420$ & $15 / 05 / 2014$ & BCELB061-19 & MT456438 \\
\hline & JSTR01297_0102 & $\mathrm{R} 012970102$ & adult $(\widehat{\partial})$ & France & Sainte-Croix-de-Quintillargue & $43.775419 / 3.907606$ & $23 / 04 / 2006$ & BCELB062-19 & MT456439 \\
\hline & JSTR01302_0101 & R013020101 & adult $(\precsim)$ & France & Assay & $47.062201 / 0.270064$ & $12 / 05 / 2006$ & BCELB063-19 & MT456440 \\
\hline & JSTR03882_0101 & R038820101 & adult $(\hat{\sigma})$ & Italy & Tricase & $39.945573 / 18.352039$ & $17 / 11 / 2016$ & BCELB071-19 & MT456444 \\
\hline & JSTR03888_0101 & R038880101 & adult $(\precsim)$ & Italy & Cascina Litta & $40.091173 / 18.167204$ & $16 / 11 / 2016$ & BCELB072-19 & MT456443 \\
\hline & JSTR04562_0101 & R045620101 & adult $(ð)$ & Spain & Aguillar-de-Segarra & $41.738710 / 1.610790$ & $6 / 9 / 2017$ & BCELB073-19 & MT456442 \\
\hline
\end{tabular}


Table 1 (continued).

\begin{tabular}{|c|c|c|c|c|c|c|c|c|c|}
\hline Species & Specimens & $\begin{array}{l}\text { Phylogenetic } \\
\text { tree codes }\end{array}$ & $\begin{array}{l}\text { Life stage } \\
\quad(\operatorname{sex})\end{array}$ & Country & Locality & $\begin{array}{l}\text { Geographic coordinates } \\
\text { Lon./Lat., decimal degr. }\end{array}$ & $\begin{array}{l}\text { Sampling } \\
\text { date }\end{array}$ & BOLD ID & $\begin{array}{r}\text { GenBank } \\
\text { Access. no. }\end{array}$ \\
\hline \multirow{44}{*}{$\begin{array}{l}\text { Oulema } \\
\text { melanopus } \\
\text { (L., 1758) }\end{array}$} & JDCH00001_0202 & - & adult $(\partial)$ & France & Berstett & $48.682758 / 7.649488$ & $07 / 07 / 2014$ & BCELB141-19 & MT456518 \\
\hline & JDCH00002_0202 & - & adult $\left({ }^{\lambda}\right)$ & France & Mirecourt & $48.286220 / 6.106403$ & $25 / 03 / 2014$ & BCELB142-19 & MT456517 \\
\hline & JDCH00003_0202 & - & adult $(\overbrace{}^{\lambda})$ & France & Gannat & $46.100167 / 3.199103$ & $24 / 04 / 2014$ & BCELB143-19 & MT456516 \\
\hline & JDCH00003_0203 & - & adult $\left(\delta^{-}\right)$ & France & Gannat & $46.100167 / 3.199103$ & $24 / 04 / 2014$ & BCELB144-19 & MT456515 \\
\hline & JDCH00005_0202 & - & adult $\left({ }^{\lambda}\right)$ & France & Treffendel & $48.037544 /-1.983165$ & $06 / 06 / 2014$ & BCELB145-19 & MT456514 \\
\hline & JDCH00006_0202 & - & adult $(\overbrace{}^{\top})$ & France & Saint-Aubin-du-Désert & $48.322195 /-0.203475$ & $15 / 05 / 2014$ & BCELB146-19 & MT456513 \\
\hline & JDCH00007_0202 & - & adult $(\hat{O})$ & France & Cossé-le-Vivien & $47.947795 /-0.921883$ & $15 / 05 / 2014$ & BCELB147-19 & MT456512 \\
\hline & JDCH00012_0202 & - & adult $\left(\delta^{\lambda}\right)$ & France & Bruxerolles & $46.611001 / 0.386810$ & $18 / 05 / 2014$ & BCELB148-19 & MT456511 \\
\hline & JDCH00013_0202 & - & adult $(\hat{O})$ & France & Mouzeuil-Saint-Martin & $46.465235 /-0.983798$ & $15 / 06 / 2014$ & BCELB149-19 & MT456510 \\
\hline & JDCH00014_0202 & - & adult $\left({ }^{\lambda}\right)$ & France & Saint-Priest & $45.687722 / 4.967944$ & $30 / 06 / 2014$ & BCELB150-19 & MT456509 \\
\hline & JDCH00015_0202 & - & adult $\left({ }^{\lambda}\right)$ & France & Orléans & - & $15 / 06 / 2013$ & BCELB151-19 & MT456508 \\
\hline & JDCH00016_0202 & - & adult $(\hat{\jmath})$ & France & Parcieux & $45.917306 / 4.835083$ & $07 / 07 / 2014$ & BCELB152-19 & MT456507 \\
\hline & JDCH00019_0202 & - & adult $(\widehat{\partial})$ & France & Neuville-Vitasse & $50.255900 / 2.809400$ & $23 / 07 / 2014$ & BCELB153-19 & MT456506 \\
\hline & JDCH00019_0203 & - & adult $(\overbrace{}^{\top})$ & France & Neuville-Vitasse & $50.255900 / 2.809400$ & $23 / 07 / 2014$ & BCELB154-19 & MT456505 \\
\hline & JDCH00020_0202 & - & adult $\left({ }^{\lambda}\right)$ & France & Verchain-Maugré & $50.266552 / 3.474370$ & $19 / 04 / 2015$ & BCELB156-19 & MT456503 \\
\hline & JDCH00024_0202 & - & adult $\left(\partial^{\lambda}\right)$ & France & Ladon & $48.004543 / 2.533953$ & $05 / 07 / 2015$ & BCELB157-19 & MT456502 \\
\hline & JDCH00025_0202 & - & adult $\left(0^{1}\right)$ & France & Montargis & $47.995572 / 2.730073$ & $12 / 07 / 2015$ & BCELB158-19 & MT456501 \\
\hline & JDCH00025_0203 & - & adult $(\overbrace{}^{\lambda})$ & France & Montargis & $47.995572 / 2.730073$ & $12 / 07 / 2015$ & BCELB159-19 & MT456520 \\
\hline & JSTR00657_0102 & - & adult $(\overbrace{}^{\lambda})$ & France & Clapiers & $43.650652 / 3.873110$ & $16 / 04 / 2014$ & BCELB155-19 & MT456504 \\
\hline & JDCH00001_0201 & H000010201 & adult $(ð)$ & France & Berstett & $48.682758 / 7.649488$ & $07 / 07 / 2014$ & BCELB160-19 & MT456499 \\
\hline & JDCH00002_0201 & H000020201 & adult $(\AA)$ & France & Mirecourt & $48.286220 / 6.106403$ & $25 / 03 / 2014$ & BCELB161-19 & MT456500 \\
\hline & JDCH00003_0201 & H000030201 & adult $(ð)$ & France & Gannat & $46.100167 / 3.199103$ & $24 / 04 / 2014$ & BCELB162-19 & MT456519 \\
\hline & JDCH00004_0201 & H000040201 & adult $(\widehat{\jmath})$ & France & Houville-la-Branche & $48.447991 / 1.625547$ & $18 / 07 / 2014$ & BCELB163-19 & MT456521 \\
\hline & JDCH00005_0201 & H000050201 & adult $(ð)$ & France & Treffendel & $48.037544 /-1.983165$ & $06 / 06 / 2014$ & BCELB164-19 & MT456522 \\
\hline & JDCH00006_0201 & H000060201 & adult $(\widehat{\jmath})$ & France & Saint-Aubin-du-Désert & $48.322195 /-0.203475$ & $15 / 05 / 2014$ & BCELB165-19 & MT456480 \\
\hline & JDCH00007_0201 & H000070201 & adult $(\hat{O})$ & France & Cossé-le-Vivien & $47.947795 /-0.921883$ & $15 / 05 / 2014$ & BCELB166-19 & MT456523 \\
\hline & JDCH00009_0201 & H000090201 & adult $(\widehat{\jmath})$ & France & Querrieu & $49.945034 / 2.424406$ & $25 / 07 / 2014$ & BCELB167-19 & MT456481 \\
\hline & JDCH00012_0201 & H000120201 & adult $(\gtrsim)$ & France & Bruxerolles & $46.611001 / 0.386810$ & $18 / 05 / 2014$ & BCELB168-19 & MT456482 \\
\hline & JDCH00013_0201 & H000130201 & adult $(ð)$ & France & Mouzeuil-Saint-Martin & $46.465235 /-0.983798$ & $15 / 06 / 2014$ & BCELB169-19 & MT456483 \\
\hline & JDCH00014_0201 & H000140201 & adult $(\gtrsim)$ & France & Saint-Priest & $45.687722 / 4.967944$ & $30 / 06 / 2014$ & BCELB170-19 & MT456484 \\
\hline & JDCH00015_0201 & H000150201 & adult $(\AA)$ & France & Orléans & - & $15 / 06 / 2013$ & BCELB171-19 & MT456485 \\
\hline & JDCH00016_0201 & H000160201 & adult $\left(\partial^{\lambda}\right)$ & France & Parcieux & $45.917306 / 4.835083$ & $07 / 07 / 2014$ & BCELB172-19 & MT456486 \\
\hline & JDCH00018_0201 & H000180201 & adult $(\AA)$ & France & Tilloy-lès-Mofflaines & $50.277301 / 2.806932$ & $15 / 05 / 2014$ & BCELB173-19 & MT456487 \\
\hline & JDCH00019_0201 & H000190201 & adult $(ð)$ & France & Neuville-Vitasse & $50.255900 / 2.809400$ & $23 / 07 / 2014$ & BCELB174-19 & MT456488 \\
\hline & JDCH00020_0201 & H000200201 & adult $(\overbrace{}^{\star})$ & France & Verchain-Maugré & $50.266552 / 3.474370$ & $19 / 04 / 2015$ & BCELB179-19 & MT456493 \\
\hline & JDCH00024_0201 & H000240201 & adult $(\gtrsim)$ & France & Ladon & $48.004543 / 2.533953$ & $05 / 07 / 2015$ & BCELB180-19 & MT456494 \\
\hline & JDCH00025_0201 & H000250201 & adult $(\AA)$ & France & Montargis & $47.995572 / 2.730073$ & $12 / 07 / 2015$ & BCELB181-19 & MT456495 \\
\hline & JSTR00656_0101 & R006560101 & adult $(\lesssim)$ & France & Clapiers & $43.658835 / 3.867831$ & $16 / 04 / 2014$ & BCELB175-19 & MT456489 \\
\hline & JSTR00666_0101 & R006660101 & adult $(\gtrsim)$ & France & Arles & $43.510278 / 4.781944$ & $27 / 04 / 2014$ & BCELB176-19 & MT456490 \\
\hline & JSTR00769_0101 & R007690101 & adult $(\gtrsim)$ & France & Le-Bouchet-Saint-Nicolas & $44.900330 / 3.748820$ & $08 / 08 / 2014$ & BCELB177-19 & MT456491 \\
\hline & JSTR01303_0101 & R013030101 & adult $(\partial)$ & France & La-Roche-Clermault & $47.139333 / 0.228444$ & 08/07/2005 & BCELB178-19 & MT456492 \\
\hline & JSTR04448_0101 & R044480101 & adult $(\AA)$ & Spain & Maçanet de la Selva & $41.762700 / 2.758420$ & $5 / 28 / 2017$ & BCELB182-19 & MT456496 \\
\hline & JSTR04454_0101 & R044540101 & adult $(\gtrsim)$ & Spain & Tordera & $41.712840 / 2.686380$ & $5 / 28 / 2017$ & BCELB183-19 & MT456497 \\
\hline & JSTR04552_0101 & R045520101 & adult $(ð)$ & Spain & Villafranca de Ebro & $41.540200 /-0.565540$ & $6 / 8 / 2017$ & BCELB184-19 & MT456498 \\
\hline \multirow{31}{*}{$\begin{array}{l}\text { Oulema obscura } \\
\text { (Stephens, 1831) }\end{array}$} & JDCH00001_0102 & - & adult $\left({ }^{\top}\right)$ & France & Berstett & $48.682758 / 7.649488$ & $07 / 07 / 2014$ & BCELB074-19 & MT456542 \\
\hline & JDCH00001_0103 & - & adult $(ð)$ & France & Berstett & $48.682758 / 7.649488$ & $07 / 07 / 2014$ & BCELB075-19 & MT456543 \\
\hline & JDCH00002_0102 & - & adult $\left({ }^{\top}\right)$ & France & Mirecourt & $48.286220 / 6.106403$ & $25 / 03 / 2014$ & BCELB076-19 & MT456544 \\
\hline & JDCH00002_0103 & - & adult $\left(\delta^{\top}\right)$ & France & Mirecourt & $48.286220 / 6.106403$ & $25 / 03 / 2014$ & BCELB077-19 & MT456545 \\
\hline & JDCH00003_0102 & - & adult $\left(\partial^{\lambda}\right)$ & France & Gannat & $46.100167 / 3.199103$ & $24 / 04 / 2014$ & BCELB078-19 & MT456536 \\
\hline & JDCH00003_0103 & - & adult $(\hat{\jmath})$ & France & Gannat & $46.100167 / 3.199103$ & $24 / 04 / 2014$ & BCELB079-19 & MT456535 \\
\hline & JDCH00004_0102 & - & adult $(\overbrace{}^{\lambda})$ & France & Houville-la-Branche & $48.447991 / 1.625547$ & $18 / 07 / 2014$ & BCELB080-19 & MT456534 \\
\hline & JDCH00005_0102 & - & adult $(\hat{\jmath})$ & France & Treffendel & $48.037544 /-1.983165$ & $06 / 06 / 2014$ & BCELB081-19 & MT456533 \\
\hline & JDCH00005_0103 & - & adult $\left(\hat{O}^{1}\right)$ & France & Treffendel & $48.037544 /-1.983165$ & $06 / 06 / 2014$ & BCELB082-19 & MT456546 \\
\hline & JDCH00006_0102 & - & adult $(\hat{\jmath})$ & France & Saint-Aubin-du-Désert & $48.322195 /-0.203475$ & $15 / 05 / 2014$ & BCELB083-19 & MT456547 \\
\hline & JDCH00007_0102 & - & adult $(\widehat{\partial})$ & France & Cossé-le-Vivien & $47.947795 /-0.921883$ & $15 / 05 / 2014$ & BCELB084-19 & MT456548 \\
\hline & JDCH00009_0102 & - & adult $(\overbrace{}^{\lambda})$ & France & Querrieu & $49.945034 / 2.424406$ & $25 / 07 / 2014$ & BCELB085-19 & MT456549 \\
\hline & JDCH00009_0103 & - & adult $(\overbrace{}^{\lambda})$ & France & Querrieu & $49.945034 / 2.424406$ & $25 / 07 / 2014$ & BCELB086-19 & MT456550 \\
\hline & JDCH00010_0102 & - & adult $(\overbrace{}^{\lambda})$ & France & Thiverval-Grignon & $48.846643 / 1.951940$ & $01 / 06 / 2014$ & BCELB087-19 & MT456551 \\
\hline & JDCH00010_0103 & - & adult $\left(0^{2}\right)$ & France & Thiverval-Grignon & $48.846643 / 1.951940$ & $01 / 06 / 2014$ & BCELB088-19 & MT456552 \\
\hline & JDCH00012_0102 & - & adult $(+)$ & France & Bruxerolles & $46.611001 / 0.386810$ & $18 / 05 / 2014$ & BCELB089-19 & MT456553 \\
\hline & JDCH00020_0102 & - & adult $(+$ ( $)$ & France & Verchain-Maugré & $50.266552 / 3.474370$ & $19 / 04 / 2015$ & BCELB095-19 & MT456559 \\
\hline & JDCH00024_0102 & - & adult $(+)$ & France & Ladon & $48.004543 / 2.533953$ & $05 / 05 / 2015$ & BCELB096-19 & MT456560 \\
\hline & JSTR01259_0102 & - & adult $(\overbrace{}^{\top})$ & France & Lorgies & $50.554260 / 2.793420$ & $15 / 05 / 2014$ & BCELB090-19 & MT456554 \\
\hline & JSTR01260_0101 & - & adult $(\overbrace{}^{-})$ & France & Neuville-Vitasse & $50.255900 / 2.809390$ & $15 / 05 / 2014$ & BCELB091-19 & MT456555 \\
\hline & JSTR01265_0101 & - & adult $(+)$ & France & Tilloy-lès-Mofflaines & $50.283450 / 2.813050$ & $15 / 05 / 2014$ & BCELB092-19 & MT456556 \\
\hline & JSTR01265_0102 & - & adult $(\overbrace{}^{-})$ & France & Tilloy-lès-Mofflaines & $50.283450 / 2.813050$ & $15 / 05 / 2014$ & BCELB093-19 & MT456557 \\
\hline & JSTR01272_0102 & - & adult $(\hat{0})$ & France & Saint-Laurent-de-Lin & $47.511404 / 0.271791$ & $22 / 07 / 2014$ & BCELB094-19 & MT456558 \\
\hline & JDCH00001_0101 & H000010101 & adult $(\hat{\jmath})$ & France & Berstett & $48.682758 / 7.649488$ & $07 / 07 / 2014$ & BCELB097-19 & MT456561 \\
\hline & JDCH00002_0101 & H000020101 & adult $(\delta)$ & France & Mirecourt & $48.286220 / 6.106403$ & $25 / 03 / 2014$ & BCELB098-19 & MT456562 \\
\hline & JDCH00003_0101 & H000030101 & adult $(\AA)$ & France & Gannat & $46.100167 / 3.199103$ & $24 / 04 / 2014$ & BCELB099-19 & MT456563 \\
\hline & JDCH00004_0101 & H000040101 & adult $(\AA)$ & France & Houville-la-Branche & $48.447991 / 1.625547$ & $18 / 07 / 2014$ & BCELB100-19 & MT456564 \\
\hline & JDCH00005_0101 & H000050101 & adult $(\gtrsim)$ & France & Treffendel & $48.037544 /-1.983165$ & $06 / 06 / 2014$ & BCELB101-19 & MT456565 \\
\hline & JDCH00006_0101 & H000060101 & adult $(ð)$ & France & Saint-Aubin-du-Désert & $48.322195 /-0.203475$ & $15 / 05 / 2014$ & BCELB102-19 & MT456566 \\
\hline & JDCH00007_0101 & H000070101 & adult $\left(\delta^{\lambda}\right)$ & France & Cossé-le-Vivien & $47.947795 /-0.921883$ & $15 / 05 / 2014$ & BCELB103-19 & MT456524 \\
\hline & JDCH00008_0102 & $\mathrm{H} 000080102$ & adult $\left(0^{\pi}\right)$ & France & Beugnâtre & $50.129035 / 2.874760$ & $14 / 05 / 2014$ & BCELB104-19 & MT456525 \\
\hline
\end{tabular}


Table 1 (continued)

\begin{tabular}{|c|c|c|c|c|c|c|c|c|c|}
\hline Species & Specimens & $\begin{array}{l}\text { Phylogenetic } \\
\text { tree codes }\end{array}$ & $\begin{array}{l}\text { Life stage } \\
\quad(\text { sex })\end{array}$ & Country & Locality & $\begin{array}{l}\text { Geographic coordinates } \\
\text { Lon./Lat., decimal degr. }\end{array}$ & $\begin{array}{l}\text { Sampling } \\
\text { date }\end{array}$ & BOLD ID & $\begin{array}{r}\text { GenBank } \\
\text { Access no. }\end{array}$ \\
\hline & JDCH00009_0101 & H000090101 & adult $(\precsim)$ & France & Querrieu & $49.945034 / 2.424406$ & $25 / 07 / 2014$ & BCELB105-19 & MT456526 \\
\hline & JDCH00010_0101 & H000100101 & adult $(\not)$ & France & Thiverval-Grignon & $48.846643 / 1.951940$ & $01 / 06 / 2014$ & BCELB106-19 & MT456527 \\
\hline & JDCH00011_0101 & H000110101 & adult $(\delta)$ & France & Tilloy-lès-Mofflaines & $50.277301 / 2.806932$ & $15 / 05 / 2014$ & BCELB107-19 & MT456528 \\
\hline & JDCH00012_0101 & H000120101 & adult $(+)$ & France & Bruxerolles & $46.611001 / 0.386810$ & $18 / 05 / 2014$ & BCELB108-19 & MT456529 \\
\hline & JDCH00020_0101 & H000200101 & adult (우) & France & Verchain-Maugré & $50.266552 / 3.474370$ & $19 / 04 / 2015$ & BCELB114-19 & MT456539 \\
\hline & JDCH00024_0101 & Н000240101 & adult $(\delta)$ & France & Ladon & $48.004543 / 2.533953$ & $05 / 05 / 2015$ & BCELB115-19 & MT456540 \\
\hline & JDCH00025_0101 & H000250101 & adult $(ð)$ & France & Montargis & $47.995572 / 2.730073$ & $12 / 07 / 2015$ & BCELB116-19 & MT456541 \\
\hline & JSTR01259_0101 & R012590101 & adult $(\delta)$ & France & Lorgies & $50.554260 / 2.793420$ & $15 / 05 / 2014$ & BCELB109-19 & MT456530 \\
\hline & JSTR01260_0102 & R012600102 & adult $(+(+)$ & France & Neuville-Vitasse & $50.255900 / 2.809390$ & $15 / 05 / 2014$ & BCELB110-19 & MT456531 \\
\hline & JSTR01262_0102 & R012620102 & adult $(+$ ( $)$ & France & Tilloy-lès-Mofflaines & $50.277510 / 2.799190$ & $15 / 05 / 2014$ & BCELB111-19 & MT456532 \\
\hline & JSTR01272_0101 & R012720101 & adult $(\precsim)$ & France & Saint-Laurent-de-Lin & $47.511404 / 0.271791$ & $22 / 07 / 2014$ & BCELB112-19 & MT456537 \\
\hline & JSTR01299_0101 & R012990101 & adult $(+\circ)$ & France & Chambray-lès-Tour & $47.339980 / 0.722099$ & $16 / 05 / 2006$ & BCELB113-19 & MT456538 \\
\hline \multirow{24}{*}{$\begin{array}{l}\text { "Oulema group } \\
\text { melanopus" }\end{array}$} & EPIE00642_0103 & - & larva & France & Beyssenac & $45.411704 / 1.324250$ & $07 / 06 / 2008$ & BCELB122-19 & MT456426 \\
\hline & EPIE00642_0104 & - & larva & France & Beyssenac & $45.411704 / 1.324250$ & $07 / 06 / 2008$ & BCELB123-19 & MT456425 \\
\hline & EPIE00642_0105 & - & larva & France & Beyssenac & $45.411704 / 1.324250$ & $07 / 06 / 2008$ & BCELB124-19 & MT456424 \\
\hline & EPIE00642_0106 & - & larva & France & Beyssenac & $45.411704 / 1.324250$ & $07 / 06 / 2008$ & BCELB125-19 & MT456423 \\
\hline & JSTR00656_0103 & - & adult $(+\circ)$ & France & Clapiers & $43.658835 / 3.867831$ & $16 / 04 / 2014$ & BCELB117-19 & MT456431 \\
\hline & JSTR00657_0101 & - & adult (우) & France & Clapiers & $43.650652 / 3.873110$ & $16 / 04 / 2014$ & BCELB118-19 & MT456430 \\
\hline & JSTR00657_0103 & - & adult (ㅇ) & France & Clapiers & $43.650652 / 3.873110$ & $16 / 04 / 2014$ & - BCELB119-19 & MT456429 \\
\hline & JSTR01263_0101 & - & adult (ㅇ) & France & Tilloy-lès-Mofflaines & $50.277510 / 2.799190$ & $15 / 05 / 2014$ & BCELB120-19 & MT456428 \\
\hline & JSTR01264_0101 & - & adult $(+$ ( $)$ & France & Tilloy-lès-Mofflaines & $50.283450 / 2.813050$ & $15 / 05 / 2014$ & BCELB121-19 & MT456427 \\
\hline & JSTR03884_0101 & - & adult $(q)$ & Italy & Cascina Litta & $40.091173 / 18.167204$ & $16 / 11 / 2016$ & BCELB126-19 & MT456422 \\
\hline & CCOC02368_0101 & $\mathrm{C} 023680101$ & larva & France & Mer & $47.721926 / 1.500711$ & $15 / 05 / 2007$ & BCELB127-19 & MT456421 \\
\hline & EPIE00642_0101 & E006420101 & larva & France & Beyssenac & $45.411704 / 1.324250$ & $07 / 06 / 2008$ & BCELB136-19 & MT456412 \\
\hline & EPIE00642_0102 & E006420102 & larva & France & Beyssenac & $45.411704 / 1.324250$ & 07/06/2008 & BCELB137-19 & MT456411 \\
\hline & JSTR00656_0102 & R006560102 & adult $(+$ ( $)$ & France & Clapiers & $43.658835 / 3.867831$ & $16 / 04 / 2014$ & BCELB129-19 & MT456419 \\
\hline & JSTR00666_0103 & R006660103 & adult $($ (ㅇ) & France & Arles & $43.510278 / 4.781944$ & $27 / 04 / 2014$ & BCELB130-19 & MT456418 \\
\hline & JSTR00669_0801 & R006690801 & adult (우) & France & Combaillaux & $43.666567 / 3.785286$ & $18 / 05 / 2014$ & BCELB128-19 & MT456420 \\
\hline & JSTR01258_0102 & R012580102 & adult (우) & France & Lorgies & $50.554260 / 2.793420$ & $15 / 05 / 2014$ & BCELB131-19 & MT456417 \\
\hline & JSTR01261_0102 & R012610102 & adult (우) & France & Neuville-Vitasse & $50.255900 / 2.809390$ & $15 / 05 / 2014$ & BCELB132-19 & MT456416 \\
\hline & JSTR01264_0102 & $\mathrm{R} 012640102$ & adult $(+$ ( $)$ & France & Tilloy-lès-Mofflaines & $50.283450 / 2.813050$ & $15 / 05 / 2014$ & BCELB133-19 & MT456415 \\
\hline & JSTR01297_0101 & R012970101 & adult (우) & France & Sainte-Croix-de-Quintillargue & $43.775419 / 3.907606$ & $23 / 04 / 2006$ & BCELB134-19 & MT456414 \\
\hline & JSTR01301_0101 & $\mathrm{R} 013010101$ & adult (우) & france & Preuilly-sur-Claise & $46.854377 / 0.928917$ & $17 / 05 / 2006$ & BCELB135-19 & MT456413 \\
\hline & JSTR03883_0101 & R038830101 & adult (우) & Italy & Collemeto & $40.203419 / 18.093920$ & $16 / 11 / 2016$ & BCELB138-19 & MT456410 \\
\hline & JSTR03885_0101 & R038850101 & adult (우) & Italy & Galugnano & $40.249590 / 18.229184$ & $16 / 11 / 2016$ & BCELB139-19 & MT456408 \\
\hline & JSTR03886_0101 & R038860101 & adult (우) & Italy & Collemeto & $40.203419 / 18.093920$ & $11 / 16 / 2016$ & BCELB140-19 & MT456409 \\
\hline $\begin{array}{l}\text { Epitrix pubesce } \\
\text { (Koch, 1803) }\end{array}$ & 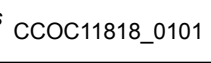 & ( & It & ance & La Ville-aux-Dames & $47.394035 / 0.770874$ & $6 / 4 / 2011$ & BCELB185-19 & MT456398 \\
\hline
\end{tabular}

using Geneious v4.6.2 (Drummond et al., 2010). Sequences were aligned with default ClustalW parameters (1.81) (Thompson et al., 1997). Consensus sequences were translated to amino acids using MEGA 7 software (Kumar et al., 2016) to detect frameshift mutations and premature stop-codons, which may have indicated the presence of pseudogenes or contaminations. Voucher specimens and associated DNA are preserved in the INRAE collections of the CBGP (Centre de Biologie pour la Gestion des Populations, Montferrier-sur-Lez, France). All sequences were deposited in Barcode of Life Data System (BOLD; Ratnasingham \& Hebert, 2007; www.boldsystems.org) and GenBank (NCBI). Our sequence dataset is available in BOLD (through the DOI dx.doi.org/10.5883/DS-BCOUL) and NCBI, accession numbers are provided in Table 1.

Pairwise nucleotide sequence divergences were calculated using the Kimura 2-parameter model of substitution (Kimura, 1980) in MEGA 7 software (Kumar et al., 2016) and the "pairwise-deletion" option. A preliminary phylogenetic tree of the genus Oulema, including all the specimens sequenced, was reconstructed based on the $C O I$ sequences. For a clearer view of the inferred tree, only the sequences of one or two specimens per locality are presented in Fig. 3 (specimens not highlighted in Table 1). We reconstructed a tree (Fig. 3) including the standard barcode COI solely for specimens reliably identified to species (only dissected males for the melanopus/duftschmidi complex) and the sequences published by Bezděk \& Baselga (2015). Phylogenetic analyses were conducted using the Maximum Likelihood (ML) method and the SMS model (Smart Model Selection)
(Lefort et al., 2017) in Phyml 3.0 software (Guindon et al., 2010). The aLRT approximation (approximate likelihood ratio test) was used to calculate the bootstrapping values for each node (Anisimova, 2006) (5000 replicates). The resulting phylogenetic trees were edited using iTOL software (Letunic \& Bork, 2016).

\section{RESULTS}

\section{Analysis of newly obtained sequences}

The barcode fragment was successfully amplified for all specimens, regardless of sex or stage. In total, 185 specimens belonging to 12 species were sequenced (Table 1). The observed minimum interspecific genetic distance between species of Chrysomelidae in the neighbouring genera of Oulema based on COI ranged from 10.2 to $19.3 \%$ (Table 3 ). In the genus Oulema, O. obscura showed a minimum divergence of $18.5 \%$ with Oulema melanopus and Oulema duftschmidi. O. melanopus showed a minimum divergence of $3.1 \%$ with $O$. duftschmidi, its closest relative. Between genera of the Criocerinae, the minimum divergence varied from $16.5 \%$ between Lema and Oulema to $21.8 \%$ between Crioceris and Oulema. Epitrix had a minimum distance from the Criocerinae species tested ranging from 22.2 to $26.2 \%$.

The intraspecific divergence levels ranged from 0 to $0.9 \%$ (mean $0.19 \% \pm 0.06 \%$ ) for $O$. melanopus, from 0 to 


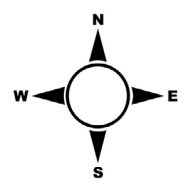

- Oulema duftschmidi male

$\star$ Oulema melanopus male

Sampling of both species
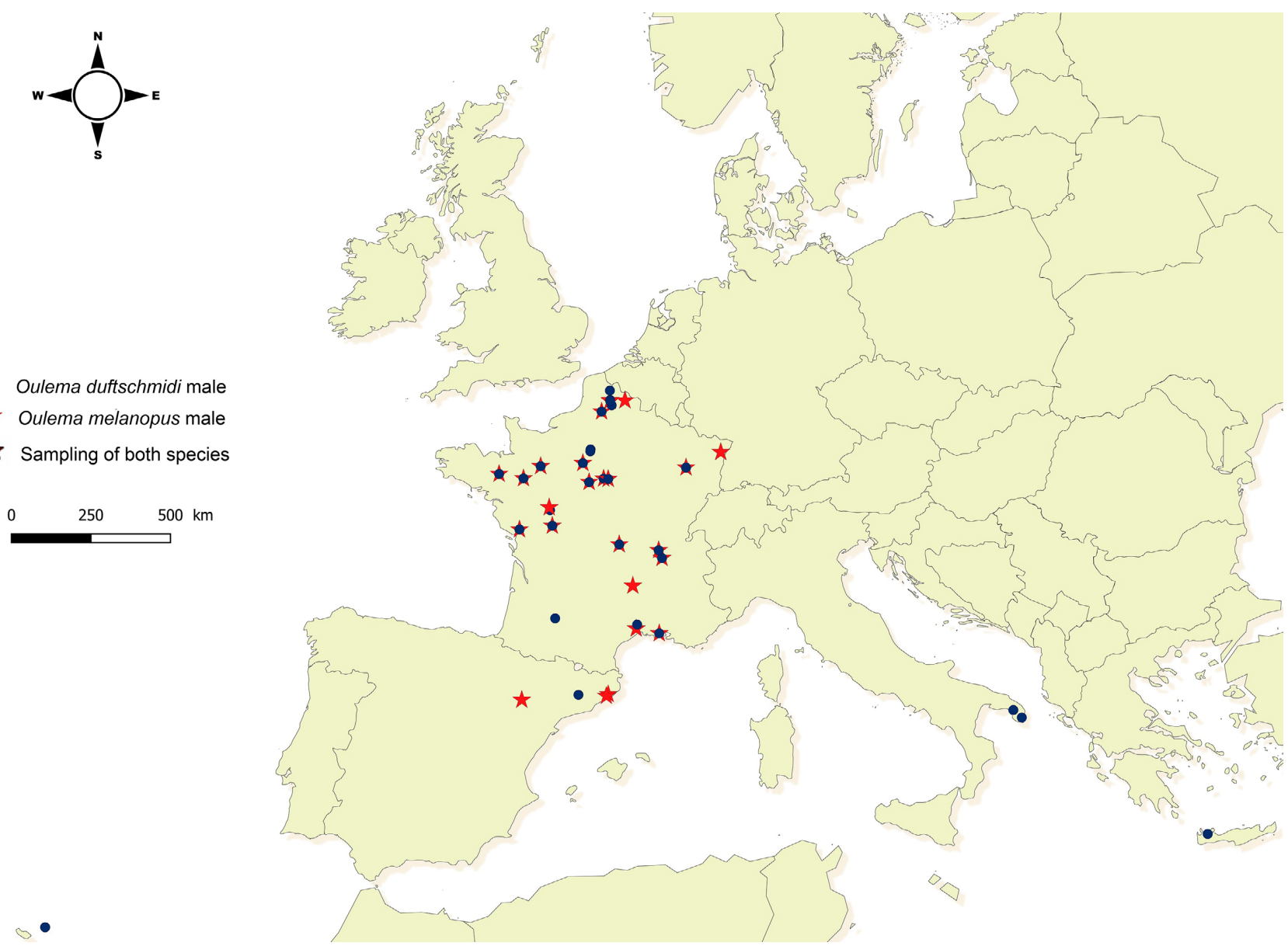

Fig. 1. Map showing the locations of the sites where males of Oulema melanopus (44 specimens) and O. duftschmidi (48 specimens) were collected. J. Leroy Design [QGIS Software version 2.18.12 (QGIS, 2016), Mapping Holdings Association: UE (Burke, 2012) and GREAT (UMS 2414 RIATE, 2018)].

Table 2. Mixture of PCR primers used in this study (based on Cruaud et al., 2010 and Germain et al., 2013). M13 tails (Ivanova et al., 2007) were used.

\begin{tabular}{ll}
\hline Name of the primer & Sequence 5'-3' of the primer \\
\hline Forward & TGTAAAACGACGGCCAGTTTTCAACWAATCATAAAGATATTGG \\
LCO1490puc_t1 & TGTAAAACGACGGCCAGTTTTCAACTAAYCATAARGATATYGG \\
LCO1490Hem1_t1 & \\
Reverse & CAGGAAACAGCTATGACTAAACTTCWGGRTGWCCAAARAATCA \\
HCO2198puc_t1 & CAGGAAACAGCTATGACTAAACYTCAGGATGACCAAAAAACA \\
HCO2198Hem2_t1 & CAGGAAACAGCTATGACTAAACYTCDGGATGBCCAAARAATCA \\
HCO2198Hem1_t1 &
\end{tabular}

$1.7 \%$ (mean $0.57 \% \pm 0.14 \%$ ) for $O$. duftschmidi and from 0 to $0.5 \%$ (mean $0.09 \% \pm 0.05 \%$ ) for $O$. obscura (Table 4).

\section{Phylogenetic reconstruction}

The substitution models selected by PhyML 3.0 as the most appropriate were the GTR $+\mathrm{G}+\mathrm{I}$ model for the tree in Fig. 3 ( $\mathrm{AIC}=10403,93488)$. That tree is based on both our sequences and those published by Bezděk \& Baselga (2015). The genus Oulema is monophyletic (support $83.26 \%$ ) with two sister clades. One (support 91.40\%) contains $O$. obscura and $O$. hoffmannseggii from Spain (as in the phylogenetic tree of Bezděk \& Baselga, 2015, in which, however, the latter species is paraphyletic); $O$. obscura contains two sister groups, one comprising specimens from the Iberian Peninsula and the other from France and the Czech Republic. The other clade containing the $O$. melanopus complex (including the 5 species recognized by Bezděk \& Baselga, 2015) is highly supported (99.42\%), but its internal relationships are problematic. O. duftschmidi forms a well-supported clade ${ }^{*}(91.36 \%)$ which, however,

* Among the dissected males, only one mismatched specimen (JSTR02905_0101 from Alentejo, Portugal, morphologically clearly belonging to $O$. duftschmidi) was nested with specimens of $O$. melanopus. This could be a labelling error or contamination (see Discussion); therefore the specimen was removed from the final analyses, from the trees in Figs 3 and S1 and consequently from sequences deposited in Bold (Table 1). 

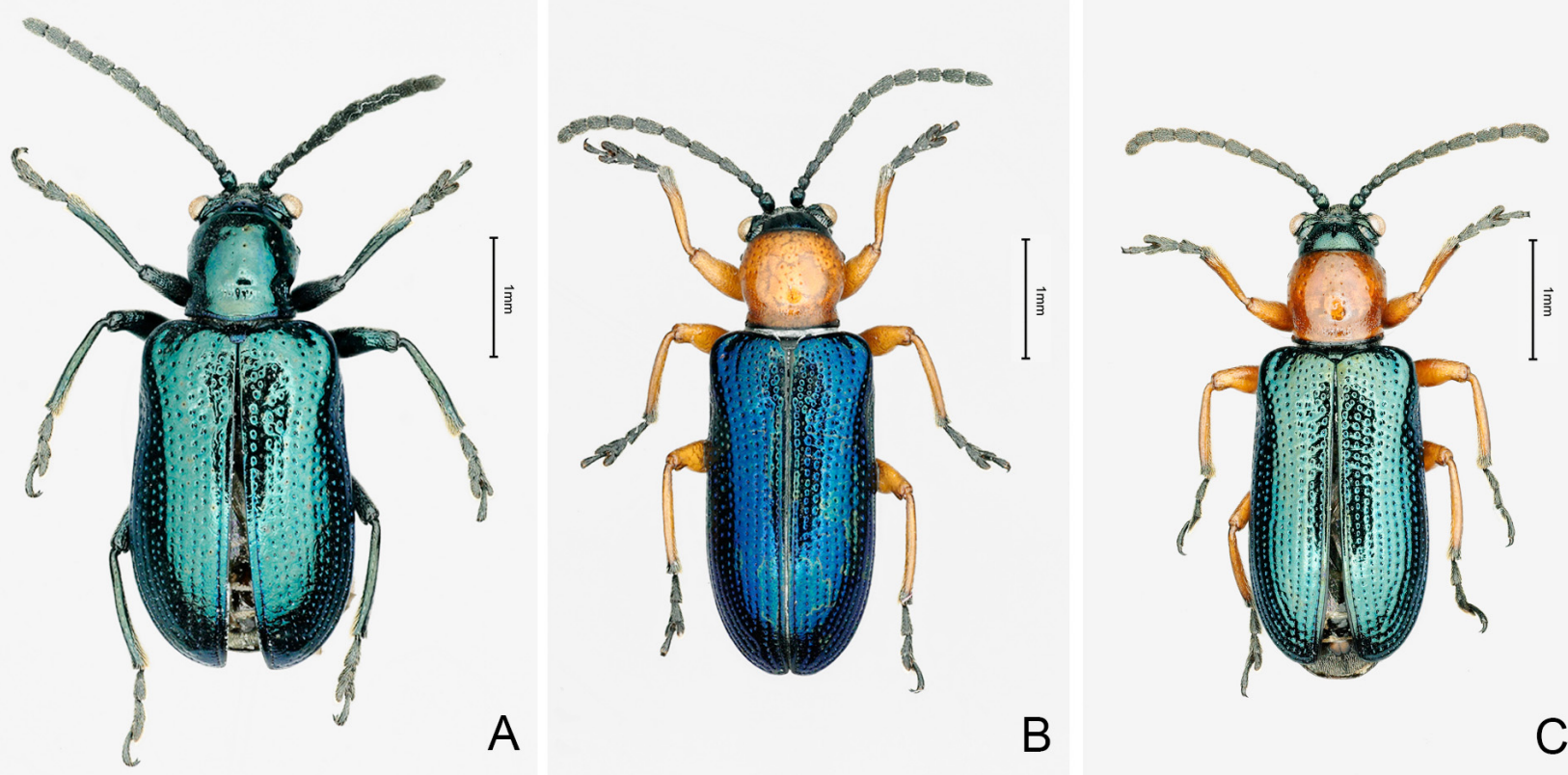

A

B
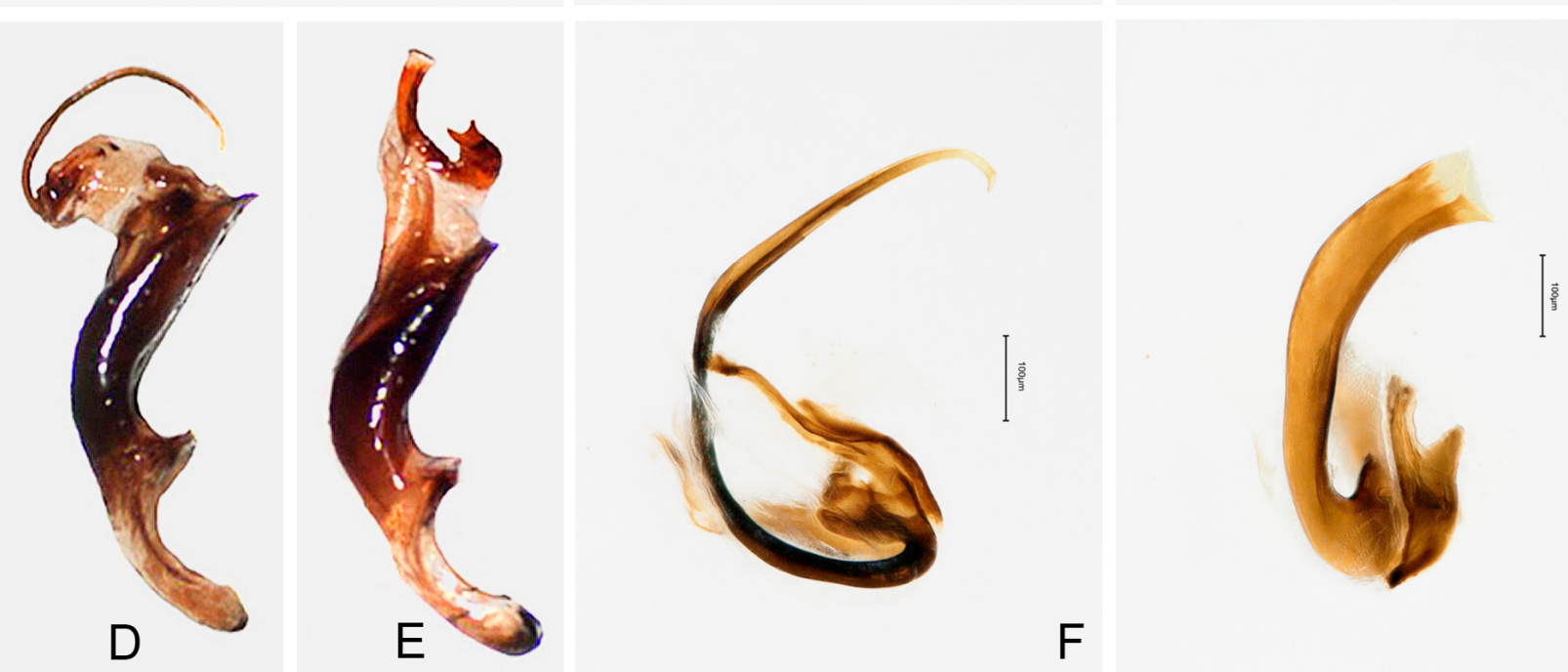

$\mathrm{F}$

Fig. 2. Habitus and male genitalia of Oulema spp. A - O. obscura (specimen JSTR1259_0101). B - O. duftschmidi (specimen JSTR0666_0102). C - O. melanopus (specimen JSTR00769_0101). D - O. duftschmidi male aedeagus in lateral view with flagellum extracted. $\bar{E}-$ O. melanopus, idem. F - O. duftschmidi flagellum (specimen JSTR01302_0101). G - O. melanopus, idem (specimen CCOC11910_010, not sequenced). Photograhs: J.-C. Streito, except D and E, which are from Bukejs \& Ferenca, 2010.

includes also the single available sequence of $O$. mauro and one specimen from Morocco identified as O. melanopus (again similar to the tree in Bezděk \& Baselga, 2015), whereas the other specimens (O. rufocyanea, O. verae and all remaining $O$. melanopus) form a markedly paraphyletic cluster and the latter two species (represented by more than one specimen) are both polyphyletic. In particular, five specimens from Galicia (NW Spain) identified as $O$. melanopus stand out and form a sister clade to $O$. rufocyanea (as in Bezděk \& Baselga, 2015).

If we exclude the sequences of Bezděk \& Baselga (2015), the resulting tree (not shown because it was essentially similar to the present Fig. S1) is congruent with morphological identifications and all species are monophyletic. The three cereal pest species had support of $100 \%$ for O. obscura, $65.94 \%$ for O. melanopus and $95.72 \%$ for $O$. duftschmidi.
When we included the unidentified specimens of the $O$. melanopus complex from France and Italy (see Table 1) in the above analysis, they were clearly placed in one or the other species (Fig. S1).

\section{DISCUSSION}

During this study, we sequenced 184 specimens of 11 species of Criocerinae in four genera (Crioceris, Lema, Lilioceris and Oulema) with a view to testing the possibility of routinely using the standard barcode COI for highthroughput and reliable identification of the three species of Oulema of agronomic interest, O. obscura, O. melanopus and $O$. duftschmidi. We included European specimens (O. duftschmidi was also available from Madeira) of 9 out of 28 species and subspecies of Criocerinae known to occur in Europe, and added specimens of 2 more Lema species from the Mascarenes. Combining our sequences with 


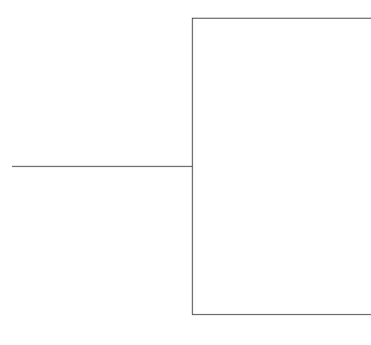

Fig. 3. Phylogenetic tree constructed using the Maximum Likelihood method (ML) and the COI gene sequences of our identified specimens of Oulema melanopus (25), Oulema duftschmidi (31), Oulema obscura (20) and 10 other species of Chrysomelidae (15), including Epitrix pubescens (in bold) used to root the tree, and the 30 sequences (also in bold) published by Bezdĕk \& Baselga (2015), giving a total of 122 sequences. Each colour corresponds to a morphologically identified species. The sequence code, identity and geographical origin are given for each specimen. The bootstrap values located at branch nodes are percentages and only those greater than $64 \%$ are presented (over 5,000 replicates). The country name is coded according to ISO 3166-1 alpha-2 (ISO 3166, 2016).

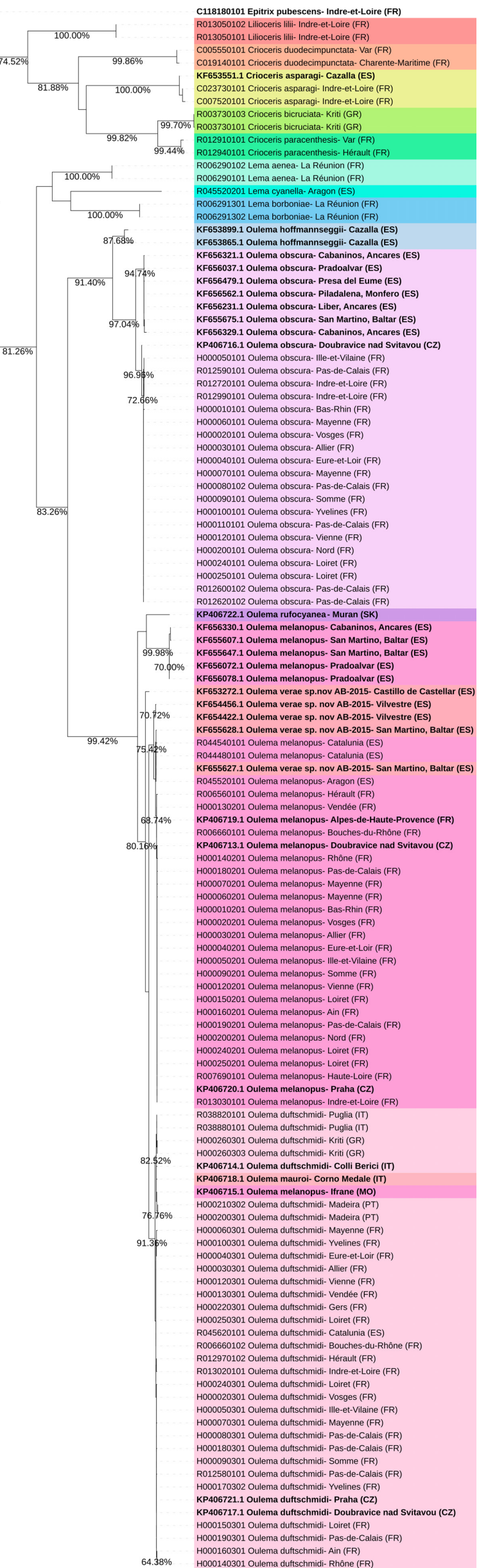


Table 3. Kimura two-parameter pairwise distance values between species (interspecific divergence). In each box the first line gives the average value and the second the minimum value. The estimated standard errors (SE) are indicated in red above the diagonal. The blue boxes show the values of distances between genera and the yellow boxes the values within the genus. 161 sequences of $O$. duftschmidi (48) and $O$. melanopus (44) males and other species of Chrysomelidae (69) were included in this analysis. Sequences from Bezdĕk \& Baselga (2015) are not included.

\begin{tabular}{|c|c|c|c|c|c|c|c|c|c|c|c|c|c|}
\hline & & $A$ & $\mathrm{~B}$ & $\mathrm{C}$ & $\mathrm{D}$ & $E$ & $\mathrm{~F}$ & $G$ & $\mathrm{H}$ & 1 & $\mathrm{~J}$ & $\mathrm{~K}$ & $\mathrm{~L}$ \\
\hline$A$ & Lilioceris lilii & & 0.020 & 0.019 & 0.018 & 0.021 & 0.019 & 0.019 & 0.020 & 0.020 & 0.020 & 0.021 & 0.020 \\
\hline B & Crioceris asparagi & $\begin{array}{c}0.218 \\
{[0,217]}\end{array}$ & & 0.018 & 0.018 & 0.017 & 0.019 & 0.020 & 0.019 & 0.021 & 0.021 & 0.021 & 0.023 \\
\hline C & Crioceris bicruciata & $\begin{array}{c}0.202 \\
{[0,202]}\end{array}$ & $\begin{array}{c}0.180 \\
{[0,176]}\end{array}$ & & 0.013 & 0.018 & 0.020 & 0.020 & 0.020 & 0.021 & 0.019 & 0.020 & 0.022 \\
\hline D & Crioceris paracenthesis & $\begin{array}{c}0.189 \\
{[0,186]}\end{array}$ & $\begin{array}{c}0.191 \\
{[0,186]}\end{array}$ & $\begin{array}{c}0.104 \\
{[0,102]}\end{array}$ & & 0.019 & 0.022 & 0.021 & 0.020 & 0.021 & 0.019 & 0.020 & 0.021 \\
\hline$E$ & $\begin{array}{l}\text { Crioceris } \\
\text { duodecimpunctata }\end{array}$ & $\begin{array}{c}0.237 \\
{[0,224]}\end{array}$ & $\begin{array}{c}0.184 \\
{[0,179]}\end{array}$ & $\begin{array}{c}0.209 \\
{[0,184]}\end{array}$ & $\begin{array}{c}0.207 \\
{[0,193]}\end{array}$ & & 0.019 & 0.020 & 0.021 & 0.020 & 0.020 & 0.021 & 0.021 \\
\hline $\mathrm{F}$ & Lema aenea & $\begin{array}{c}0.204 \\
{[0,204]}\end{array}$ & $\begin{array}{c}0.197 \\
{[0,193]}\end{array}$ & $\begin{array}{c}0.218 \\
{[0,217]}\end{array}$ & $\begin{array}{c}0.242 \\
{[0,238]}\end{array}$ & $\begin{array}{c}0.204 \\
{[0,198]}\end{array}$ & & 0.015 & 0.018 & 0.017 & 0.018 & 0.018 & 0.021 \\
\hline G & Lema borboniae & $\begin{array}{c}0.208 \\
{[0,208]}\end{array}$ & $\begin{array}{c}0.226 \\
{[0,223]}\end{array}$ & $\begin{array}{c}0.219 \\
{[0,219]}\end{array}$ & $\begin{array}{c}0.230 \\
{[0,226]}\end{array}$ & $\begin{array}{c}0.229 \\
{[0,221]}\end{array}$ & $\begin{array}{c}0.136 \\
{[0,136]}\end{array}$ & & 0.017 & 0.018 & 0.019 & 0.019 & 0.020 \\
\hline $\mathrm{H}$ & Lema cyanella & $\begin{array}{c}0.214 \\
{[0,214]}\end{array}$ & $\begin{array}{c}0.211 \\
{[0,205]}\end{array}$ & $\begin{array}{c}0.231 \\
{[0,230]}\end{array}$ & $\begin{array}{c}0.227 \\
{[0,223]}\end{array}$ & $\begin{array}{c}0.228 \\
{[0,222]}\end{array}$ & $\begin{array}{c}0.169 \\
{[0,169]}\end{array}$ & $\begin{array}{c}0.159 \\
{[0,159]}\end{array}$ & & 0.019 & 0.020 & 0.021 & 0.020 \\
\hline I & Oulema obscura & $\begin{array}{c}0.206 \\
{[0,204]}\end{array}$ & $\begin{array}{c}0.240 \\
{[0,232]}\end{array}$ & $\begin{array}{c}0.242 \\
{[0,240]}\end{array}$ & $\begin{array}{c}0.229 \\
{[0,226]}\end{array}$ & $\begin{array}{c}0.222 \\
{[0,214]}\end{array}$ & $\begin{array}{c}0.165 \\
{[0,161]}\end{array}$ & $\begin{array}{c}0.166 \\
{[0,165]}\end{array}$ & $\begin{array}{c}0.190 \\
{[0,188]}\end{array}$ & & 0.020 & 0.019 & 0.022 \\
\hline$J$ & Oulema melanopus & $\begin{array}{c}0.214 \\
{[0,208]}\end{array}$ & $\begin{array}{c}0.225 \\
{[0,217]}\end{array}$ & $\begin{array}{c}0.220 \\
{[0,215]}\end{array}$ & $\begin{array}{c}0.218 \\
{[0,211]}\end{array}$ & $\begin{array}{c}0.223 \\
{[0,212]}\end{array}$ & $\begin{array}{c}0.175 \\
{[0,170]}\end{array}$ & $\begin{array}{c}0.190 \\
{[0,186]}\end{array}$ & $\begin{array}{c}0.197 \\
{[0,192]}\end{array}$ & $\begin{array}{c}0.193 \\
{[0,185]}\end{array}$ & & 0.007 & 0.022 \\
\hline K & Oulema duftschmidi & $\begin{array}{c}0.222 \\
{[0,218]}\end{array}$ & $\begin{array}{c}0.232 \\
{[0,224]}\end{array}$ & $\begin{array}{c}0.226 \\
{[0,220]}\end{array}$ & $\begin{array}{c}0.235 \\
{[0,230]}\end{array}$ & $\begin{array}{c}0.237 \\
{[0,229]}\end{array}$ & $\begin{array}{c}0.176 \\
{[0,171]}\end{array}$ & $\begin{array}{c}0.191 \\
{[0,186]}\end{array}$ & $\begin{array}{c}0.206 \\
{[0,198]}\end{array}$ & $\begin{array}{c}0.192 \\
{[0,185]}\end{array}$ & $\begin{array}{c}0.037 \\
{[0,031]}\end{array}$ & & 0.021 \\
\hline L & $\begin{array}{l}\text { Epitrix pubescens } \\
\text { (Galerucinae) - outgroup }\end{array}$ & $\begin{array}{c}0.235 \\
{[0,235]}\end{array}$ & $\begin{array}{c}0.263 \\
{[0,262]}\end{array}$ & $\begin{array}{c}0.260 \\
{[0,260]}\end{array}$ & $\begin{array}{c}0.252 \\
{[0,250]}\end{array}$ & $\begin{array}{c}0.240 \\
{[0,206]}\end{array}$ & $\begin{array}{c}0.222 \\
{[0,222]}\end{array}$ & $\begin{array}{c}0.234 \\
{[0,234]}\end{array}$ & $\begin{array}{c}0.224 \\
{[0,224]}\end{array}$ & $\begin{array}{c}0.245 \\
{[0,243]}\end{array}$ & $\begin{array}{c}0.233 \\
{[0,230]}\end{array}$ & $\begin{array}{c}0.234 \\
{[0,228]}\end{array}$ & \\
\hline
\end{tabular}

those of Bezděk \& Baselga (2015), 7 European species of Oulema (from the 11 known in Europe) were documented. Our results confirm those of Kubisz et al. (2012) and show that the standard DNA barcode can reliably differentiate between most European species of Criocerinae, but confirm also the results of Bezděk \& Baselga (2015) that this marker cannot differentiate between all species of the genus Oulema. A complex of species occurs in the Mediterranean basin including at least $O$. melanopus, $O$. verae, O. duftschmidi, O. mauroi and possibly some undescribed cryptic species, which cannot be reliably distinguished by the standard barcode. In this study, sampling and marker selection were used to address agronomic questions. The lack of material coming from non-cultivated Mediterranean ecosystems prevented us addressing the problem of the species occurring around the Mediterranean basin where

Table 4. Kimura two-parameter pairwise average distance values within the species studied (intraspecific divergence) ( $d$ - average; max - maximum). The estimated standard errors (SE) are given. $161 \mathrm{se}-$ quences of $O$. duftschmidi (48) and $O$. melanopus (44) males and other species of Chrysomelidae (69) were included in this analysis. Distances could not be estimated for species with only one individual (Lema cyanella and Epitrix pubescens). Sequences from Bezděk \& Baselga (2015) are not included.

\begin{tabular}{lcccc}
\hline & $\mathrm{d}$ & $\mathrm{SE}$ & $\mathrm{max}$ \\
\cline { 2 - 5 } \cline { 4 - 5 } Oulema melanopus & 0.0019 & & 0.0006 & 0.009 \\
Oulema duftschmidi & 0.0057 & & 0.0014 & 0.017 \\
Oulema obscura & 0.0009 & & 0.0005 & 0.005 \\
Crioceris duodecimpunctata & 0.0183 & 0.0042 & 0.035 \\
Crioceris asparagi & 0.0037 & 0.0015 & 0.009 \\
Crioceris bicruciata & 0.0015 & 0.0015 & 0.002 \\
Crioceris paracenthesis & 0.0038 & 0.0016 & 0.008 \\
Lema aenea & 0 & 0 & $\mathrm{n} / \mathrm{c}$ \\
Lema borboniae & 0 & 0 & 0 \\
Lilioceris lilii & 0 & 0 & 0 \\
\hline
\end{tabular}

much more extensive sampling and the use of other markers and other methods would be needed to clarify the taxonomy of Oulema.

\section{Distinguishing Oulema obscura from the O. melanopus complex}

While adult specimens of $O$. obscura can be easily distinguished from those of the melanopus group on the basis of their general coloration (body entirely blue versus red pronotum and legs, respectively) (Fig. 2a versus $2 b, c)$, the use of DNA barcodes for species identification can also be used to reliably identify the immature stages of these species. The sequence of the COI gene tested makes it possible to distinguish this species from the entire melanopus complex which includes the other two Oulema cereal pests (O. melanopus and $O$. duftschmidi). The minimum divergence of $18.5 \%$ between $O$. obscura and O. melanopus $/ O$. duftschmidi is rather high and comparable to distances recorded between species in different genera in the same subfamily, such as Crioceris and Lema (Table 3).

\section{Distinguishing between 0 . melanopus and O. duftschmidi}

The minimum interspecific divergence of $3.1 \%$ between specimens of $O$. melanopus and $O$. duftschmidi (Table 3) means that these sibling species are more closely related than all the other species studied, which is consistent with their similar morphology and biology. In addition, such a value is congruent with what is reported for other sibling species in the family Chrysomelidae (Cognato, 2006). However, we recorded lower genetic distances between specimens of $O$. duftschmidi from very distant populations (North of France and the Italian province of Puglia) or isolated populations (such as those in Madeira and Crete), than between specimens of $O$. melanopus and $O$. 
duftschmidi from France, despite being collected together, in the same place and at the same time (Tables 3 and 4).

Lastly, the interspecific percentage divergences (Table 3) were well above the maximum percentages of intraspecific divergence recorded (Table 4) and there was no overlap between the intra- and interspecific distances of $O$. melanopus and $O$. duftschmidi. Consequently, this argues in favour of a clear genetic differentiation of the sibling species $O$. duftschmidi and $O$. melanopus, which is supported by the phylogenetic trees (Figs 3 and S1). Of the 92 males studied, only one (JSTR02905_0101, a male specimen of O. duftschmidi from Alentejo, Portugal), was placed in a cluster that does not correspond with the species identification based on the morphology of its genitalia. A posteriori examination of the preserved adult and its dissected genitalia definitively excluded misidentification. However, we cannot exclude an error in tube labelling during handling or contamination. Wherever possible, we deliberately selected specimens of the two species for our dataset that were collected on the same day at the same location (see Table 1), to maximize the chances of recording potential hybridisation. Apart from this specimen, for which it was not possible to rule out a handling error, no other individual was incorrectly assigned in our data set. A second case of a mismatch between molecular and morphological identification was that of the Moroccan specimen (PK406715.1), which was genetically assigned to $O$. duftschmidi, whereas it was identified as $O$. melanopus by Bezděk \& Baselga (2015). These authors (pers. com.) suspected that there was an undescribed cryptic species in Morocco to which this specimen belonged. Indeed, they noted differences between the genitalia of this specimen and typical specimens of $O$. melanopus with which it was tentatively identified. The method we used (extraction, amplification and sequencing of a gene) enabled correct assignment of males previously identified on the basis of dissected genitalia. The unidentified females and larvae that we tested were also unambiguously assigned to one of the two taxonomic groups. It would be interesting to test the method on a larger number of specimens in order to check whether introgression has occurred and assess its percentage of occurrence. Breeding tests would also be required to test this hypothesis.

\section{Distinguishing other species in the melanopus complex and their intraspecific differences}

The intraspecific diversity of specimens from the Mediterranean basin, especially those from the Iberian Peninsula, was greater than that of the French and Czech specimens (Fig. 3). This increase in genetic diversity with increase in the geographical coverage is documented (Bergsten et al., 2012) and due to the presence of Mediterranean glacial refugia and their associated biological diversity (Hewitt, 2001). Currently the data from the Mediterranean areas is limited and more extensive sampling could provide additional insights into the biological or biogeographical processes that resulted in the present diversity.

The other issue is distinguishing between the cereal pests and the rarer or more localized species that are described in the genus Oulema. Phylogenetic relationships between the five species of the melanopus group have been studied by Bezděk \& Baselga (2015) based on the COI gene. They conclude that the relationships between the different species in this group were not well resolved on the basis of this gene. The results we obtained by combining their work with ours are slightly more optimistic at least in the possibility of distinguishing $O$. duftschmidi from any European specimens currently being morphologically identified as $O$. melanopus and support the idea that these two groups are genetically well separated. In Fig. 3, O. mauroi and the Moroccan specimen KF406717.1 presently identified as $O$. melanopus are nested within $O$. duftschmidi, and the sequences of $O$. verae are intermixed with the remaining specimens identified as $O$. melanopus. We did not undertake a morphological study of $O$. mauroi and $O$. verae, which are rare in collections, but according to Bezděk \& Baselga (2015), the morphological differences between $O$. verae/O. melanopus and $O$. mauroi/O. duftschmidi are much more marked than the differences between $O$. melanopus and $O$. duftschmidi. O. rufocyanea, also a member of the $O$. melanopus complex, is clustered with a subgroup of Spanish specimens from Galicia (Bold BIN ACJ0414) morphologically identified as $O$. melanopus. The population from Morocco remains to be studied. It is possible that the standard COI barcode is not suitable for discriminating between species in the Mediterranean area. The possibility of introgressions having occurred will have to be explored along with increased sampling and use of more relevant molecular markers, especially nuclear markers.

\section{Other European species of Oulema}

Oulema obscura and O. hoffmannseggii form a sister group to the $O$. melanopus complex (as in Bezděk \& Baselga, 2015). A more comprehensive sampling of the under-represented species and the addition of the 4 remaining species (O. erichsonii, O. septentrionis, O. tristis and $O$. magistrettiorum) should further improve our understanding of this genus. The fact that the specimens of $O$. melanopus from Galicia differed from the others also indicates that there may be additional cryptic diversity in the genus Oulema, warranting further studies using an integrated approach.

\section{Oulema sequences available in BOLD and reliability of the barcoding identification of European species}

BOLD system (Ratnasingham \& Hebert, 2007) currently (November 2020) contains 436 Oulema sequences forming 15 Barcode Index Numbers (BINs). Three BINs contain most of the sequences:

AAK5928: 178 sequences of which 155 are identified as $O$. melanopus, two as $O$. erichsonii, and the remaining sequences are not identified to species level. This BIN includes 44 sequences from the present study identified as $O$. melanopus.

AAO0694: 107 sequences of which 83 are identified as $O$. duftschmidi, one as $O$. mauroi and two as O. melanopus. This BIN includes 48 sequences from the present study, all identified as $O$. duftschmidi. 
AAN1559: 77 sequences of which 76 are identified as O. obscura (or its synonym O. gallaeciana) and one is unidentified.

Surprisingly, $O$. verae is not included in BOLD while $O$. mauroi from the same study (Bezděk \& Baselga, 2015) is included.

The remaining $12 \mathrm{BINs}$ are represented by a limited number of sequences (one to ten). Several species names are associated with several BINs: $O$. erichsonii (four different BINs among which is AAK5928); O. duftschmidi (AAO0694 and ADK1309 for one Indian sequence); $O$. obscura under the name O. gallaeciana (AAN1559 and ABW1444 for the 7 sequences from Spain, Galicia, see Fig. 3); O. hoffmannseggii (ABV0207 and ADU7791 for two sequences from Spain); O. melanopus (AAK5928 and four other BINs for sequences from different European and non-European countries, of which ABW1460 contains among other the 5 sequences from Spain, Galicia, sister to $O$. rufocyanea in our Fig. 3). That sequence of $O$. rufocyanea (KP406722.1) is associated with a unique BIN (ACJ0414). 44 sequences were not identified to species and 46 were not associated to a BIN due to their poor quality, insufficient length, etc.

BOLD provides the state-of-the-art barcoding information of the genus Oulema and highlights the need of clarification of the taxonomy in this group. The association of one species with several BINs and conversely several species within the same BIN may not be only due to misidentification. We cannot exclude some cryptic species such as the 7 specimens identified as $O$. gallaeciana from Spain that form a separate BIN. At the present state of knowledge, the use of the database for routine identification of Oulema can only be considered in a limited geographic context, keeping in mind possible misidentifications and the partly unresolved taxonomy. For that reason we chose to compare our results only to sequences resulting from a taxonomic study (Bezděk \& Baselga, 2015).

\section{CONCLUSION}

Bergsten et al. (2012) highlighted that limited sampling, and thus a restricted set of sequences reflecting local biodiversity, improves the identification by barcoding. This is supported by our results. Depending on the geographical context and the agronomic versus natural context, the identification of Oulema species by barcoding may be more or less efficient.

This study showed that the standard COI barcode can be used to distinguish between some Oulema species, including $O$. melanopus and $O$. duftschmidi, but cannot distinguish some other species in the melanopus complex, suggesting that further analyses might be needed to validate their taxonomic status.

Very extensive sampling has been carried out recently in agricultural regions in France (Chapelin-Viscardi \& Maillet-Mezeray, 2015; Leroy \& Chapelin-Viscardi, 2018). Several thousand specimens were identified based on the shape of the male flagellum. Given the relatively clear morphological criteria that characterise $O$. mauroi and $O$. verae (Bezděk \& Baselga, 2015), it is unlikely that they would have been confused with $O$. melanopus and $O$. duftschmidi in those studies. Identification based on male genitalia, and especially flagella, tested by specialists, was validated by our study, which also confirmed the quality of the morphological identifications. These surveys provide evidence that only three species of Oulema are present in cereal crops in France: O. obscura, O. melanopus and $O$. duftschmidi. In the absence of the other species of the melanopus complex, the results obtained show that DNA barcoding is a good method for differentiating between species of the genus Oulema in cereal crops in France, regardless of the developmental stage or sex of the specimens. In order to meet the needs of plant protection professionals, the method must provide both unambiguous and reliable results. To achieve this, it will be necessary first of all to associate the reference sequences present in the database (on which the identification of sequences will be carried out) to a given geographical area and context, in our case cereal fields in mainland France.

ACKNOWLEDGEMENTS. We thank the many contributors who collected samples in the field: C. Schaub (Alsace Chamber of Agriculture), D. Marcolet (INRA Mirecourt), J. Dourlens, A. Roncier, S. Boucher (Bayer CropScience), J.-C. Lebreton (Mayenne Chamber of Agricuture), F. Couturier-Boiton (Aube Chamber of Agriculture), A. Gardarin (INRA Grignon), P. Cantot (Société Entomologique de France), D. Dentinger (Vendée Chamber of Agriculture), B. Cailleret (FREDON) and C. Cocquempot (INRA Montpellier). We are grateful to B. Gauthier and J. Fleury for assisting us in laboratory analyses at the Laboratoire d'Eco-Entomologie. Thanks to A. Bukejs for allowing us to use the photographs of Oulema aedeagi. Many thanks to P. Biggins (Cirad) who reviewed the English language of this manuscript.

\section{REFERENCES}

ACTA 2016: Guide pratique de défense des cultures. ACTA éditions, Paris, 913 pp.

Anglade P., Roehrich R., Maurin G. \& Lecigne P. 1976: Le Léma des céréales (Oulema melanopus L.), étude de sa nuisibilité en Aquitaine. - Défense Végét. 178: 85-100.

Anisimova M. \& GASCuel O. 2006: Approximate likelihood-ratio test for branches: A fast, accurate, and powerful alternative. Syst. Biol. 55: 539-552.

Balachowsky A. \& Mesnil L. 1935: Les insectes nuisibles aux plantes cultivées. Leurs mœurs, leur destruction. Établissements Busson, Paris, 1137 pp.

Bechini L., Morlacchi P. \& Baumgärtner J. 2013: Coinciding development of winter wheat and leaf beetles along an Alpine transect. - Acta Oecol. 47: 95-104.

Bergsten J., Bilton D.T., Fujisawa T., Elliott M., Monaghan M. T., Balke M., Hendrich L., Geijer J., Herrmann J., Foster G. N., Ribera I., Nilsson A.N., Barraclough T.G. \& Vogler A.P. 2012: The effect of geographical scale of sampling on DNA barcoding. - Syst. Biol. 61: 851-869.

BERTI N. 1989: Contribution à la faune de France. L'identité d'Oulema (O.) melanopus (L.) [Col. Chrysomelidae Criocerinae]. - Bull. Soc. Entomol. Fr. 94: 47-57.

BezděK J. \& Baselga A. 2015: Revision of western Palaearctic species of the Oulema melanopus group, with description of two new species from Europe (Coleoptera: Chrysomelidae: Criocerinae). - Acta Entomol. Mus. Nat. Prag. 55: 273-304. 
BEZDĚK J. \& MLEJNEK R. 2016: Icones insectorum Europae centralis. Coleoptera: Megalopodidae, Orsodacnidae, Chrysomelidae: Donaciinae, Criocerinae. - Folia Heyrovskyana (B) 27: 1-63 [in Czech and English].

BezdĚK J. \& Schмiтt M. 2017: Subfamily Criocerinae. In Löbl I. $\&$ Smetana A. (eds): Catalogue of Palaearctic Coleoptera, vol. 6, Corrigenda et Addenda. - Entomol. Bl. Col. 113: 113-135.

BonNEMAISON L. 1962: Les ennemis animaux des plantes cultivées et des forêts, tome II. Éditions Sep, Paris, 500 pp.

Bunejs A. \& Ferenca R. 2010: The first record of Oulema duftschmidi (Redtenbacher, 1874) (Coleoptera: Chrysomelidae) in the Lithuanian fauna. - Acta Zool. Lituan. 20: 229 231.

BURKE R 2012: UE. URL: http://www.arcgis.com/home/item.ht $\mathrm{ml}$ ?id=6d611 f8d87d54227b494d4c3becef6a0 (last accessed 18 Jan. 2018).

Chambon J.-P., Van Laere C., Genestier G., Pineau C. \& CocQUEMPOT C. 1983: Étude des populations d'Oulema melanopus L. et Oulema lichenis Weiss. (Coleoptères: Chrysomelidae) sur blé dans la région parisienne. - Agronomie 3: 685-690.

Chapelin-Viscardi J.-D. \& Maillet-Mezeray J. 2015: Données bio-écologiques sur deux espèces jumelles sympatriques en milieux agricoles: Oulema melanopus (L.) et $O$. duftschmidi (Redtenbacher) (Coleoptera, Chrysomelidae). — Bull. Mens. Soc. Linn. Lyon 84: 19-30.

Cognato A.I. 2006: Standard percent DNA sequence difference for insects does not predict species boundaries. - J. Econ. Entomol. 99: 1037-1045.

Cox M.L. 2000: Progress report on the Bruchidae/Chrysomelidae recording scheme. - Coleopterist 9: 65-74.

Cruaud A., Jabbour-Zahab R., Genson G., Cruaud C., Couloux A., KJellberg F., van Noort S. \& Rasplus J.-Y. 2010: Laying the foundations for a new classification of Agaonidae (Hymenoptera: Chalcidoidea), a multilocus phylogenetic approach. - Cladistics 26: 359-387.

Drummond A.J., Ashton B., Buxton S., Cheung M., Cooper A., Heled J., Kearse M., Moir R., Stones-Havas S., Sturrock S., ThIERER T. \& Wilson A. 2010: Geneious v5.5. URL: http:// www.geneious.com.

Feytaud J. 1924: Un parasite de la Miliade, Lema flavipes Suffr. - Rev. Zool. Agric. Appl. 23: 158-161.

Germain J.-F., Сhatot C., Meusnier I., Artige E., Rasplus J.-Y. \& CRuaud A. 2013: Molecular identification of Epitrix potato flea beetles (Coleoptera: Chrysomelidae) in Europe and North America. - Bull. Entomol. Res. 103: 354-362.

Guindon S., Dufayard J.-F., Lefort V., Anisimova M., HordiJk W. \& GASCUEL O. 2010: New algorithms and methods to estimate maximum-likelihood phylogenies: Assessing the performance of PhyML 3.0. - Syst. Biol. 59: 307-321.

Guppy J.-C. \& Harcourt D.G. 1978: Effects of temperature on development of the immature stages of the cereal leaf beetle, Oulema melanopus (Coleoptera: Chrysomelidae). - Can. Entomol. 110: 257-263.

Hebert P.D.N., Cywinska A. \& Ball S.L. 2003a: Biological identifications through DNA barcodes. - Proc. R. Soc. Lond. (B) 270: 313-321.

Hebert P.D.N., Ratnasingham S. \& DeWaArd J.R. 2003b: Barcoding animal life: cytochrome c oxidase subunit 1 divergences among closely related species. - Proc. R. Soc. Lond. (B) 270: S96-S99.

HewitT G.M. 2001: Speciation, hybrid zones and phylogeography - or seeing genes in space and time. - Mol. Ecol. 10: $537-549$.
Ivanova N.V., Zemlak T.S., Hanner R.H. \& Hebert P.D.N. 2007: Universal primer cocktails for fish DNA barcoding. - Mol. Ecol. Notes 7: 544-548.

Jolivet P. 1997: Biologie des Coléoptères Chrysomélides. Société Nouvelle des Éditions Boubée, Paris, 279 pp.

KimURA M. 1980: A simple method for estimating evolutionary rate of base substitutions through comparative studies of nucleotide sequences. - J. Mol. Evol. 16: 111-120.

Knechtel W.K. \& Manolache C.I. 1936: Observatii biologice asupra gândacului ovazului (Lema melanopa L.) în România. - Anal. Inst. Cercet. Agron. Român. 7: 186-208.

Kubisz D., Kajtoch Ł., Mazur M.A. \& Rizun V. 2012: Molecular barcoding for central-eastern European Crioceris leaf-beetles (Coleoptera: Chrysomelidae). - Centr. Eur. J. Biol. 7: 69-76.

Kumar S., Stecher G. \& Tamura K. 2016: Mega7: molecular evolutionary genetics analysis version 7.0 for bigger datasets. - Mol. Biol. Evol. 33: 1870-1874.

LABEYRIE V. 1963: Sous-famille des Criocerinae. In Balachowsky A.S. 1963: Entomologie appliquée à l'agriculture. Tome 1, Coléoptères, Second volume, Phytophagoidea (suite et fin). Masson et Cie, Paris, pp. 571-592.

Lefort V., Longueville J.-E. \& Gascuel O. 2017: SMS: Smart model selection in PhyML 3.0. - Mol. Biol. Evol. 34: 24222424.

Leroy J. \& Chapelin-Viscardi J.-D. 2018: Sympatrie et syntopie de deux espèces jumelles: Oulema melanopus (L., 1758) et Oulema duftschmidi (Redtenbacher, 1874) (Coleoptera: Chrysomelidae). - Entomologiste 74: 163-169.

LETUNIC I. \& BoRK P. 2016: Interactive tree of life (iTOL) v3: an online tool for the display and annotation of phylogenetic and other trees. - Nucl. Acids Res. 44: W242-W245.

Olfert O. \& Weiss R.M. 2006: Impact of climate change on potential distributions and relative abundances of Oulema melanopus, Meligethes viridescens and Ceutorhynchus obstrictus in Canada. - Agric. Ecosyst. Environ. 113: 295-301.

PÉlÉCASSIS E. 1951: Le Lema melanopa L. nuisible sur les céréales en Grèce. - Ann. Inst. Phytopathol. Benaki 5: 52.

Philips C.R., Herbert D.A., Kuhar T.P., Reisig D.D., Thomason W.E. \& MALONE S. 2011: Fifty years of cereal leaf beetle in the US: an update on its biology, management, and current research. - J. Integr. Pest Manag. 2: C1-C5.

QGiS Development Team 2016: QGIS Geographic Information System. Open Source Geospatial Foundation Project. URL: http://qgis.osgeo.org (last accessed 18 Jan. 2018).

Ratnasingham S. \& Hebert P.D.N. 2007: BOLD: The Barcode of Life Data System (www.barcodinglife.org). - Mol. Ecol. Notes 7: 355-364.

Riley E.G., Clark S.M. \& Seeno T.N. 2003: Catalog of the Leaf Beetles of America North of Mexico (Coleoptera: Megalopodidae, Orsodacnidae and Chrysomelidae, Excluding Bruchinae). Coleopterists Society, Special Publication no. 1, Sacramento, CA, $290 \mathrm{pp}$.

SAJó K. 1893: Das Getreidehähnchen (Lema melanopus L.). $-Z$. Pflanzenkrankh. 3: 129-137.

Streito J.-C., Fontaine O., Atiama M., Genson G., Pierre É., Sadeyen J., Frago E. \& Nibouche S. 2018: Présence sur l'île de la Réunion de deux espèces de punaises prédatrices potentiellement utilisables pour la lutte biologique: Orius naivashae et Cyrtopeltis callosus (Hemiptera, Anthocoridae et Miridae). - Bull. Soc. Entomol. Fr. 123: 1-128.

Thompson J.D., Gibson T.J., Plewniak F., Jeanmougin F. \& HigGINS D.G. 1997: The CLUSTAL-X windows interface: flexible strategies for multiple sequence alignment aided by quality analysis tools. — Nucl. Acids Res. 25: 4876-4882. 
UMS 2414 RIATE 2018: GREAT (Generalised Representation for European Areas and Territories). URL: http://riate.cnrs. $\mathrm{fr} /$ ?page id=153 (accessed 18 Jan. 2018).

Urquiso P. 1940: Una plaga de Lema melanopus L. en trigos de Galicia. - Bol. Patol. Veg. Entomol. Agric. 9: 263-267.

Venturi F. 1942: La Lema melanopa L. (Coleoptera: Chrysomelidae). - Redia 28: 11-88.
WarchaŁowski A. 2003: Chrysomelidae: The Leaf-beetles of Europe and the Mediterranean Area. Natura Optima Dux Foundation, Warszawa, $656 \mathrm{pp}$.

White R.E. 1981: Homonymy in Word Species Group Names of Criocerinae (Coleoptera: Chrysomelidae). U.S. Department of Agriculture, Technical Bulletin 1629, 69 pp.

Received March 24, 2020; revised and accepted July 14, 2020 Published online December 21, 2020

Supplementary Fig. S1 follows on next page. 


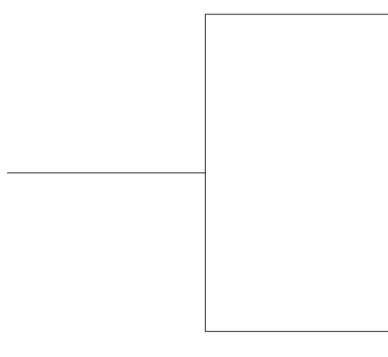

Tree scale: $0.1 \longmapsto$
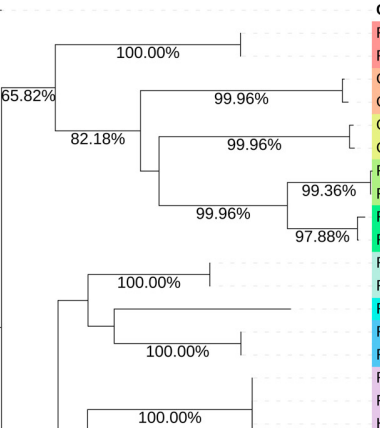

72.0

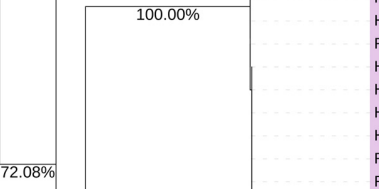

C118180101 Epitrix pubescens- Indre-et-Loire (FR)

R013050101 Lilioceris lilii- Indre-et-Loire (FR)

R013050102 Llilioceris lilili-Indre-et-Loire (FR)

C005550101 Crioceris duodecimpunctata- $\operatorname{Var}$ (FR)

rente-Maritime (FR)

C007520101 Crioceris asparagi- Indre-et-Loire (FR)

C023730101 Crioceris asparagi- Indre-et-Loire (FR)

R003730101 Crioceris bicruciata- Kriti (GR)

R003730103 Crioceris bicruciata- Kriti (GR)

R012910101 Crioceris paracenthesis- Var (FR)

R012940101 Crioceris paracenthesis- Hérault (FR)

R006290101 Lema aenea- La Réunion (FR)

R006290102 Lema aenea- La Réunion (FR)

R045520201 Lema cyanella-Aragon (ES)

R006291301 Lema borboniae- La Réunion (FR)

R006291302 Lema borboniae- La Réunion (FR)

R012720101 Oulema obscura- Indre-et-Loire (FR)

R012590101 Oulema obscura- Pas-de-Calais (FR)

H000050101 Oulema obscura- Ille-et-Vilaine (FR)

R012990101 Oulema obscura- Indre-et-Loire (FR)

H000030101 Oulema obscura-Allier (FR)

Ho00060101 Oulema obscura- Mayenne (FR)

H000020101 Oulema obscura- Vosges (FR)

H000200101 Oulema obscura- Nord (FR)

R012620102 Oulema obscura- Pas-de-Calais (FR)

R012600102 Oulema obscura- Pas-de-Calais (FR)

H000120101 Oulema obscura- Vienne (FR)

H000250101 Oulema obscura- Loiret (FR)

H000240101 Oulema obscura- Loiret (FR)

Ho00110101 Oulema obscura- Pas-de-Calais (FR)

H000100101 Oulema obscura- Yvelines (FR)

H000090101 Oulema obscura- Somme (FR)

H000080102 Oulema obscura- Pas-de-Calais (FR)

H000070101 Oulema obscura- Mayenne (FR)

H000040101 Oulema obscura- Eure-et-Loir (FR)

H000010101 Oulema obscura- Bas-Rhin (FR)

R044480101 Oulema melanopus- Catalunia (ES)

R044540101 Oulema melanopus- Catalunia (ES)

R045520101 Oulema melanopus- Aragon (ES)

R006560101 Oulema melanopus- Hérault (FP)

R006660101 Oulema melanopus- Bouches-du-Rhône (FR)

R012970101 Oulema grp melanopus- Hérault (FR)

H000130201 Oulema melanopus- Vendée (FR)

H000060201 Oulema melanopus- Mayenne (FR)

E006420102 Oulema grp melanopus- Corrèze (FR)

R006660103 Oulema grp melanopus- Bouches-du-Rhône (FR)

H000070201 Oulema melanopus- Mayenne (FR)

H000180201 Oulema melanopus- Pas-de-Calais (FR)

E006420101 Oulema grp melanopus- Corrèze (FR)

R013010101 Oulema grp melanopus- Indre-et-Loire (FR)

R012580102 Oulema grp melanopus- Pas-de-Calais (FR)

R006690801 Oulema grp melanopus- Hérault (FR)

H000250201 Oulema melanopus- Loiret (FR)

H000240201 Oulema melanopus- Loiret (FR)

H000200201 Oulema melanopus- Nord (FR)

R013030101 Oulema melanopus- Indre-et-Loire (FR)

R007690101 Oulema melanopus- Haute-Loire (FR)

Ho00190201 Oulema melanopus- Pas-de-Calais (FR)

H000160201 Oulema melanopus- Ain (FR)

H000140201 Oulema melanopus- Rhône (FR)

H000150201 Oulema melanopus- Loiret (FR)

H000120201 Oulema melanopus- Vienne (FR)

Ho00090201 Oulema melanopus- Somme (FR)

H000050201 Oulema melanopus- Ille-et-Vilaine (FR)

H000040201 Oulema melanopus- Eure-et-Loir (FR)

H000030201 Oulema melanopus- Allier (FR)

H000010201 Oulema melanopus- Bas-Rhin (FR)

Ho00020201 Oulema melanopus- Vosges (FR)

R038860101 Oulema grp melanopus- Puglia (IT)

R038860101 Oulema grp melanopus- Puglia (IT)
Ho00060301 Oulema duftschmidi- Mayenne (FR)

Ho00060301 Oulema duftschmidi- Mayenne (FR)
H000040301 Oulema duftschmidi- Eure-et-Loir (FR)

H000040301 Oulema dutschmidi- Eure-et-Loir (FR)

Ho00120301 Oulema duftschmidi- Vienne (FR)

R006660102 Oulema duftschmidi- Bouches-du-Rhône (FR)

H000130301 Oulema duftschmidi- Vendée (FR)

H000030301 Oulema duftschmidi- Allier (FR)

H0000220301 Oulema duftschmidi- Gers (FR)

R045620101 Oulema duftschmidi- Catalunia (ES)

H000250301 Oulema duftschmidi- Loiret (FR)

R006560102 Oulema grp melanopus- Hérault (FR)

R012970102 Oulema duftschmidi- Hérault (FR)

R013020101 Oulema duftschmidi- Indre-et-Loire (FR)

Ho00200301 Oulema duftschmidi- Madeira (PT)

H00020030 2 O

H000260301 Oulema duftschmidi-Kriti (GR)

H000260303 Oulema duftschmidi-Kriti (GR)

R038820101 Oulema duftschmidi- Puglia (IT)

R0388800101 Oulema duftschmidi- Puglia (IT)

R038830101 Oulema grp melanopus- Puglia (IT)

R0388850101 Oulema grp melanopus- Puglia (IT)

H000140301 Oulema duftschmidi- Phône (FP)

H000140301 Oullma dutschmidi-Rhône (FR)

H000160301 UD

R012640102 Oulema grp melanopus- Pas-de-Calais (FR)

H012640102 Oulema glp melanopus- Pas-de-Ca

R012580101 Oulema duftschmidi- Pas-de-Calais (FR)

R012610102 Oulema grp melanopus- Pas-de-Calais (FR)

H000170302 Oulema duftschmidi- Yvelines (FR)

C023680101 Oulema grp melanopus- Loir-et-Cher (FR)

C02360101 Orlema grp melanopus- Loir-et-Cher (FR)

H000150301 Oulema dutschmidi- Loiret (FR)

H0000901 Out

Ho0000301 Ous

Hoo0080301 Odlema dunchinid-Pas-de-Calais (FR)

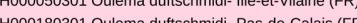

H000020301 Oulema duftschmidi- Vosges (FR)

than $64 \%$ are presented (over 5 is coded according to ISO 3166-1 alpha-2 (ISO 3166, 2016). 